Y. Miyahara

Nagoya Math. J.

Vol. 81 (1981), 177-223

\title{
INFINITE DIMENSIONAL LANGEVIN EQUATION AND FOKKER-PLANCK EQUATION
}

\author{
YOSHIO MIYAHARA
}

\section{§. Introduction}

Stochastic processes on a Hilbert space have been discussed in connection with quantum field theory, theory of partial differential equations involving random terms, filtering theory in electrical engineering and so forth, and the theory of those processes has greatly developed recently by many authors (A. B. Balakrishnan [1, 2], Yu. L. Daletskii [7], D. A. Dawson [8, 9], Z. Haba [12], R. Marcus [18], M. Yor [26]).

The most basic concept arising there is the so-called cylindrical Brownian motion, abbr. c.B.m., (see Definition 1.2). It is thought of as a natural generalization of a finite dimensional Brownian motion, and it can be formed from multi-parameter white noise as is briefly illustrated in what follows.

First we introduce a (Gaussian) white noise $\mu$ indexed by a spacetime parameter running through $D \times T$, where $D$ is a domain of the $d$ dimensional Euclidean space $\boldsymbol{R}^{d}$ and $T$ is $\boldsymbol{R}^{1}$ on which the time $t$ runs. Namely, $\mu$ is the standard Gaussian measure on $\mathscr{E}^{*}$ determined by the characteristic functional

$$
C_{\mu}(\eta)=\exp \left\{-\frac{1}{2}\|\eta\|^{2}\right\}, \quad\|\eta\|^{2}=\int_{D \times T}|\eta|^{2} d x, \quad \eta \in \mathscr{E},
$$

where $\mathscr{E}^{*}$ is the dual of $\mathscr{E}$ forming a Gelfand triple:

$$
\mathscr{E}^{*} \subset \mathscr{H}=L^{2}(D \times T) \subset \mathscr{E}^{*} .
$$

We are now given a generalized stochastic process in the sense that $\langle\eta, \omega\rangle$, $\eta \in \mathscr{E}, \omega \in \mathscr{E}^{*}$, is an ordinary random variable, where $\langle$,$\rangle is the canonical$ bilinear form connecting $\mathscr{E}$ and $\mathscr{E}^{*}$ (I.M. Gelfand and N. Ya. Vilenkin [11]). The bilinear form $\langle$,$\rangle ,extends to the case where \eta$ is of the form $\xi \otimes \chi_{[s, t]}$

Received October 25, 1979. 
in such a way that $\left\langle\xi \otimes \chi_{[s, t]}, \omega\right\rangle$ is still a random variable on $\left(\mathscr{E}^{*}, \mu\right)$. With this remark in mind, set

$$
B_{t}(\xi)=\left\langle\xi \otimes \chi_{[0 \wedge t, 0 \vee t]}, \omega\right\rangle, t \in R^{1} .
$$

Then it is a Brownian motion with parameter space $\boldsymbol{R}^{1}$ with variance $\|\xi\|_{L^{2}(D)}^{2}$. In view of this, $B_{t}(\cdot)$ is called a cylindrical Brownian motion. Stochastic integrals with respect to c.B.m. can be defined in a usual manner. Details will be discussed in $\S 1$ to some extent. It is noted that white noise is given as soon as we take the time-derivative $(d / d t) B_{t}(\cdot)$ of $B_{t}(\cdot)$, and it is indexed by $\xi$.

In $\S 2$ we shall summarize some known results, which have been obtained by the author ([19] and [20]) about stochastic differential equations on a Hilbert space. In $\S 3$ we shall focus our attention on the most basic equation

$$
d X_{t}=-\hat{\omega} X_{t} d t+d B_{t}
$$

on $H=L^{2}([0, \pi])$, where $B_{t}$ is a c.B.m. on $H$ and $\hat{\omega}=\sqrt{-\Delta}\left(\Delta=d^{2} / d \sigma^{2}\right.$ : Laplacian on $L^{2}([0, \pi])$ with Neumann boundary condition). Unfortunately the equation (1) has no solution in $H$, and so we are led to extend the state space. Put $\tilde{H}=\left\{\tilde{X} \in H ;(\tilde{X}, 1)_{H}=0\right\}$. If $\hat{\omega}$ is restricted to $\tilde{H}$, it is a strictly positive operator such that $\hat{\omega}^{-1}$ is of Hilbert-Schmidt type. We are therefore able to construct a Hilbert scale derived from $\hat{\omega}$ (for definition see Yu. L. Daletskii [7]):

$$
\tilde{H}_{\infty} \subset \cdots \subset \tilde{H}_{1} \subset \tilde{H}_{1 / 2} \subset \tilde{H} \subset \tilde{H}_{-1 / 2} \subset \tilde{H}_{-1} \subset \cdots \subset \tilde{H}_{-\infty},
$$

and hence the equation (1) is viewed as an equation on $\tilde{H}$ or on this Hilbert scale instead of on the original $H$.

We are now ready to state our main results. The equation (1) has a unique solution on $\tilde{H}_{-1 / 2}$, which comes from the discussion in $\S 2$. There exists a unique invariant probability measure $\nu$ of $\tilde{X}_{t}$ given by (1) on $\tilde{H}_{-1 / 2}$ and it is proved that the transition probability $P(t, \tilde{X}, d \tilde{Y})$ of the Markov process $\tilde{X}_{t}$ is always equivalent to $\nu(d \tilde{Y})$. As for the Radon-Nikodym derivative $P(t, \tilde{X}, \tilde{Y})=d P(t, \tilde{X}, \cdot) / d \nu(\cdot)$ we have a version, still denote by the same symbol, satisfying enough analytic properties as is prescribed by the following theorem.

THEOREM 3.4. The function $P(t, \tilde{X}, \tilde{Y})$ has the following properties:

(i) $P(t, \tilde{X}, \tilde{Y})$ is a continuous function on $(0, \infty) \times \tilde{H}_{-1 / 2} \times \tilde{H}_{-1 / 2}$. 
(ii) $P(t, \tilde{X}, \tilde{Y})=P(t, \tilde{Y}, \tilde{X})$, that is $P(t, \tilde{X}, \tilde{Y})$ is symmetric.

(iii) For fixed $t>0$ and $\tilde{X} \in \tilde{H}_{-1 / 2}, P(t, \tilde{X}, \tilde{Y})$ is an $L^{p}(d \nu)$-function of $\tilde{Y}$ for every $p>0$. But $P(t, \tilde{X}, \tilde{Y})$ is not bounded.

(iv) $P(t, \tilde{X}, \tilde{Y})$ is the fundamental solution of the following equation

$$
\begin{gathered}
\frac{\partial P(t, \tilde{X}, \tilde{Y})}{\partial t}=\frac{1}{2} \Delta_{V} P(t, \tilde{X}, \tilde{Y})-\int_{0}^{\pi} \hat{\omega} \tilde{Y}(\sigma) \frac{\delta P(t, \tilde{X}, \tilde{Y})}{\delta \tilde{Y}(\sigma)} d \sigma, \\
P(t, \tilde{X}, \tilde{Y}) \longrightarrow \delta_{\tilde{X}}(\tilde{Y}) \quad(t \downarrow 0),
\end{gathered}
$$

where $\Delta_{V}$ denotes the Volterra Laplacian and $\delta P / \delta \tilde{Y}(\sigma)$ denotes the functional derivative (see I. Kubo [16] §7 and Remark 3.1 given below), and where the precise meaning of (3.15) is that

$$
\int_{\tilde{H}_{-1 / 2}} P(t, \tilde{X}, \tilde{Y}) f(\tilde{Y}) d \nu(Y) \longrightarrow f(\tilde{X}) \quad \text { as } t \downarrow 0
$$

for any continuous bounded function $f(\tilde{Y})$ of $\tilde{H}_{-1 / 2}$.

Another main theorem is concerned with the generator $L$, associated with $\tilde{X}_{t}$, acting on the Hilbert space $L^{2}\left(\tilde{H}_{-1 / 2}, d \nu\right)$. Let $\Phi_{\left\{n_{j}\right\}}$ be a FourierHermite polynomial (see $\S 3$ ). Having extended $L$ to be a closed operator on $L^{2}(d \nu)$, we have

Theorem 3.6. (i) The functions $\left\{\Phi_{\left\{n_{j}\right\}}(\tilde{Y})\right\}, n_{j}=0,1, \cdots, \sum n_{j}<\infty$, satisfy the following formula

$$
L \Phi_{\left\{n_{j}\right\}}(\tilde{Y})=-\left(\sum_{j} j n_{j}\right) \Phi_{\left\{n_{j}\right\}}(\tilde{Y}) .
$$

The operator $L$, acting on $L^{2}(d \nu)$, is non-positive definite and self-adjoint.

(ii) The function $P(t, \tilde{X}, \tilde{Y})$ satisfies the equation

$$
\frac{d P}{d t}(t, \tilde{X}, \tilde{Y})=L P(t, \tilde{X}, \tilde{Y}), \quad t>0, \text { for fixed } \tilde{X}
$$

These results are interesting in connection with the theory of string model as well (C. Rebbi [22], M. Kaku and K. Kikkawa [15], and Z. Haba and J. Lukierski [13]). In fact, the equation (3.14) is similar to (3.4) of [15] and the formula (3.13), which is given in $\S 3$, is similar to (4.2) of [15] in appearance. Actually, our approach was inspired by these works.

In the last part of $\S 3$, we shall briefly discuss equations of the form $d \tilde{X}_{t}=-\hat{\omega} \tilde{X}_{t} d t+B d B_{t}$, where $B$ is a bounded linear operator on $\tilde{H}$.

As we have mentioned above, c.B.m. $B_{t}(\cdot)$ is derived from a white noise $\mu$. We therefore expect some intimate connections between the space 
$L^{2}\left(\mathscr{E}^{*} \rightarrow K\right)$ arising from white noise $\mu, K$ being a Hilbert space, and stochastic integrals with respect to $B_{t}(\cdot)$. In fact, we shall be able to see them in $\S 4$ in the Wiener's direct sum decomposition of $L^{2}\left(\mathscr{E}^{*} \rightarrow K\right)$ and its representation formula by means of multiple Wiener integrals or of iterated stochastic integrals w.r.t. c.B.m. In the case of $K=\boldsymbol{R}^{1}$, the above results are known (see T. Hida [14]), however our generalization, where $K$ is infinite dimensional, requires to establish some basic techniques such as iterated stochastic integrals, the correspondence between $\sigma_{2}\left(L^{2}\left((D \times T)^{n}\right)\right.$, $K)$ (= a Hilbert space consisting of all Hilbert-Schmidt operators from $L^{2}\left((D \times T)^{n}\right)$ to $\left.K\right)$ and $L^{2}\left((D \times T)^{n} \rightarrow K\right)$, and etc.

We then come to another important topic to discuss the equation of the form

$$
d X_{t}=-\hat{\omega} X_{t} d t+X_{t} \cdot d B_{t},
$$

where $X_{t} \cdot$ is a multiplicative operator defined by $\left(X_{t} \cdot h\right)(\sigma)=X_{t}(\sigma) \times h(\sigma)$. Similar equations, but somewhat different equations of this type have been investigated by many authors (D.A. Dawson [8, 9, 10], A.V. Balakrishnan [2], A. Shimizu [23]), however we can show that (2) can be dealt with in line with the analysis on $L^{2}\left(\mathscr{E}^{*} \rightarrow K\right)$.

The equation (2), describing actual phenomenon, is itself interesting, but unfortunately it has no solution on $H$, and even not on a Hilbert scale. We shall therefore consider in $\S 5$ a modified equation of the form

$$
d X_{t}=-\hat{\omega} X_{t} d t+\left(\int_{0}^{\pi} \Gamma\left(\sigma, \sigma^{\prime}\right) X_{t}\left(\sigma^{\prime}\right) d \sigma^{\prime}\right) \cdot d B_{t}
$$

where $\Gamma($,$) is an element of H \times H_{1}$. This equation does have a unique solution in $H_{-1}$ (Theorem 5.1). Our discussions in $\S 5$ are based on the theory developed in $\S 4$, and the main results are Theorem 5.2 and Theorem 5.5. The former (Theorem 5.2) gives a system of equations in terms of the kernels of the integral representation of $X_{t}$, while the latter (Theorem 5.5) gives a system of ordinary differential equations on $H_{-1}$ which is proved to be equivalent to (3).

The author wishes to thank Professor T. Hida for his valuable suggestions and kind encouragement in preparing the manuscript.

\section{$\S 1$. Multi-parameter white noise and cylindrical Brownian motion}

Let $D$ be a domain of the $d$-dimensional Euclidean space $\boldsymbol{R}^{d}$. Let $H$ be a real Hilbert space $L^{2}(D)$ and let $\mathscr{H}$ be a real Hilbert space $L^{2}(D \times T)$, 
where $T=R^{1}$. We start with a Gelfand triple

$$
\mathscr{E} \subset \mathscr{H}=L^{2}(D \times T) \subset \mathscr{E}^{*},
$$

where $\mathscr{E}$ is a nuclear space and $\mathscr{E}^{*}$ is the dual space of $\mathscr{E}$. Given a characteristic function

$$
C_{\mu}(\eta)=\exp \left\{-\frac{1}{2}\|\eta\|^{2}\right\}, \quad\|\eta\|^{2}=\int_{D \times T}|\eta|^{2} d x
$$

we are given a probability space $\left(\mathscr{E}^{*}, \mathscr{B}, \mu\right)$ such that

$$
C_{\mu}(\eta)=\int_{\delta^{*}} e^{i\langle\eta, \omega\rangle} d \mu(\omega)
$$

where $\langle$,$\rangle is the canonical bilinear form connecting \mathscr{E}$ and $\mathscr{E} *$.

Definition 1.1. The probability space $\left(\mathscr{E}^{*}, \mathscr{B}, \mu\right)$ is called a (Gaussian) white noise on $H$ or a (Gaussian) white noise with parameter space $D \times T$.

In this paper we adopt the white noise space $\left(\mathscr{E}^{*}, \mathscr{B}, \mu\right)$ as the basic probability space, and we denote by $\mathscr{B}_{t}$ the $\sigma$-field generated by $\{\langle\eta, \omega\rangle$; $\left.\eta \in \mathscr{E}, \omega \in \mathscr{E}^{*}, \operatorname{supp}\{\eta\} \subset D \times(-\infty, t]\right\}$.

We next will give a definition of a cylindrical Brownian motion. Assume that a probability space $(\Omega, F, P)$ and an increasing family of $\sigma$-field $F_{t}, t \geqq 0, F_{t} \subset F$, are given.

Definition 1.2 (M. Yor [26]). A mapping $B_{t}(h, \omega):[0, \infty) \times H \times \Omega \rightarrow \boldsymbol{R}^{1}$ is called a cylindrical Brownian motion (abbr. c.B.m.) on $H$ if it satisfies the following conditions:

(i) $B_{0}(h, \cdot)=0$ and $B_{t}(h, \cdot)$ is $F_{t}$-adapted.

(ii) For any $h \in H, h \neq 0,(1 /\|h\|) B_{t}(h, \cdot)$ is a one-dimensional Brownian motion.

(iii) For any $t \in[0, \infty)$ and $\alpha, \beta \in R^{1}$ and $h, k \in H$, the following formula holds

$$
B_{t}(\alpha h+\beta k)=\alpha B_{t}(h)+\beta B_{t}(k), \quad(P \text {-a.s. }) .
$$

Remark 1.1. If necessary, we can take a continuous version of $B_{t}(h)$. The process $B_{t}$ can not be regarded as a process on $H$.

We will now form a c.B.m. on $H=L^{2}(D)$ from a white noise. Put $B_{t}(\xi)=\left\langle\xi \otimes \chi_{[0, t]}, \omega\right\rangle$, where $\xi \in H$ and $\chi_{[0, t]}$ is the defining function of the interval $[0, t]$ and $\omega \in \mathscr{E}^{*}$. The function $\left\langle\xi \otimes \chi_{[0, t]}, \omega\right\rangle$ is not well-defined on $\mathscr{E}^{*}$, because $\xi \otimes \chi_{[0, t]}$ may not belong to $\mathscr{E}$. But, we know that if $\eta_{j} \rightarrow$ 
$\eta$ in $L^{2}(D \times T)$, then $\left\langle\eta_{j}, \omega\right\rangle \rightarrow\langle\eta, \omega\rangle$ in $L^{2}\left(\mathscr{E}^{*}, \mu\right)$. By using this fact, we are able to define a random variable $\left\langle\xi \otimes \chi_{[0, t]}, \omega\right\rangle$ as the mean square limit of a sequence $\left\langle\eta_{j}, \omega\right\rangle, j=1,2, \cdots$, where $\eta_{j} \in \mathscr{E}$ and $\eta_{j} \rightarrow \xi \otimes \chi_{[0, t]}$. It is easy to see that the mapping $B_{t}(\xi)=\left\langle\xi \otimes \chi_{[0, t]}, \omega\right\rangle$ satisfies the conditions (i)-(iii) in Definition 1.2.

We will define stochastic integrals with respect to the c.B.m. $B_{t}$ constructed above. Let $\phi(t, \omega)$ be a $\mathscr{B}_{t}$-adapted measurable function on $[0, \infty)$ $\times \mathscr{E}^{*}$ into $H$ such that

$$
E\left[\int_{0}^{t}\|\phi(s)\|^{2} d s\right]<\infty \quad \text { for any } t>0
$$

where $E$ [ ] means the expectation with respect to $\mu$. Then a real valued martingale $\sum_{n=1}^{\infty} \int_{0}^{t}\left(\phi(s), e_{n}\right) d B_{s}\left(e_{n}\right)$ is well-defined in the ordinary sence, where $\left\{e_{n}\right\}, n=1,2, \cdots$, is an orthonormal base in $H$.

Definition 1.3. The stochastic integral $\int_{0}^{t}\left\langle\phi(s), d B_{s}\right\rangle$ of $\phi$ is the martingale given by

$$
\int_{0}^{t}\left\langle\phi(s), d B_{s}\right\rangle=\sum_{n=1}^{\infty} \int_{0}^{t}\left(\phi(s), e_{n}\right) d B_{s}\left(e_{n}\right)
$$

Remark 1.2. We can easily prove that the definition of stochastic integrals does not depend on the choice of a base $\left\{e_{n}\right\}$, and that the following equation holds:

$$
E\left[\left|\int_{0}^{t}\left\langle\phi(s), \mid d B_{s}\right\rangle\right|^{2}\right]=E\left[\int_{0}^{t} \| \phi(s)||^{2} d s\right] .
$$

Given two Hilbert spaces $H$ and $K$, we denote by $\sigma_{2}(H, K)$ the Hilbert space consisting of all Hilbert-Schmidt operators from $H$ into $K$. Let $\Phi(t, \omega)$ be a $\sigma_{2}(H, K)$-valued $\mathscr{B}_{t}$-adapted function defined on $[0, \infty) \times \mathscr{E} *$ into $\sigma_{2}(H, K)$ such that

$$
E\left[\int_{0}^{t}\|\Phi(s)\|_{\sigma_{2}(H, K)}^{2} d s\right]<\infty
$$

Then the integral

$$
\int_{0}^{t}\left\langle\Phi^{*}(s) y, d B_{s}\right\rangle, \quad \Phi^{*}(s) \text { the dual operator of } \Phi(s),
$$

is well-defined for every $y \in K$, and it is linear in $y$. 
Definition 1.4. The stochastic integral of $\Phi$ is the $K$-valued martingale $M_{t}$ which is uniquely determined by

$$
\left(y, M_{t}\right)_{K}=\int_{0}^{t}\left\langle\Phi^{*}(s) y, d B_{s}\right\rangle, \quad y \in K,
$$

and it is denoted by $\int_{0}^{t} \Phi(s) d B_{s}$.

Remark 1.3. It is easy to verify

$$
E\left[\left\|\int_{0}^{t} \Phi(s) d B_{s}\right\|^{2}\right]=E\left[\int_{0}^{t}\|\Phi(s)\|_{o_{2}(H, K)}^{2} d s\right] .
$$

\section{§2. Stochastic differential equations on a Hilbert space}

Given two real separable Hilbert spaces $H$ and $K$, we consider a stochastic integral equation on $K$

$$
X_{t}=X_{0}+\int_{0}^{t} a\left(X_{s}\right) d s+\int_{0}^{t} G\left(X_{s}\right) d B_{s},
$$

where $a$ and $G$ are mappings such that $a: K \rightarrow K, G: K \rightarrow \sigma_{2}(H, K)$, and $B_{s}$ is a c.B.m. on $H$. For simplicity, we write the equation (2.1) in the form of stochastic differential equation

$$
d X_{t}=a\left(X_{t}\right) d t+G\left(X_{t}\right) d B_{t}
$$

In the case where $K=H$ and where $a$ and $G$ have suitable properties, the equations of this type have been well investigated (e.g., M. Yor [26]). This paper deals with such equations without much restriction on $G$ but with a specific drift term. Namely, we consider the equation of the form

$$
d X_{t}=-A X_{t} d t+G\left(X_{t}\right) d B_{t}
$$

where $A$ is an unbounded linear operator with the domain $D(A)$ of dense in $K$. We will summarize the results obtained by the author in [19] and [20] for the equation (2.3).

We assume that $-A$ is the infinitesimal generator of a semi-group $\left\{T_{t}\right\}, t \geqq 0$, of class $C_{0}$ and that $G$ satisfies the following condition

$$
\|G(X)\|_{\sigma_{2}(H, K)} \leqq c_{1}+c_{2}\|X\|_{K},
$$

where $c_{1}$ and $c_{2}$ are constants. The equation (2.3) is expressed in the form

$$
X_{t}=X_{0}+\int_{0}^{t}-A X_{s} d s+\int_{0}^{t} G\left(X_{s}\right) d B_{s} .
$$


Since $A$ is unbounded, the condition $X_{s} \in D(A)$ is not always satisfied. While, by the assumptions on $-A$, the conjugate operator $-A^{*}$ of $-A$ is the infinitesimal generator of the conjugate semi-group $\left\{T_{t}^{*}\right\}$, which is of class $C_{0}$, and the domain $D\left(A^{*}\right)$ of $A^{*}$ is dense in $K$. Taking these facts into consideration, two different kinds of solutions can be defined.

Definition 2.1 ( $w$-solution). A weak solution of (2.4) is a $\mathscr{B}_{t}$-adapted process $X_{t}$ on $K$ which satisfies the following conditions:

(i ) $E\left[\int_{0}^{t}\left\|X_{t}\right\|^{2} d s\right]<\infty \quad$ for any $t \geqq 0$.

(ii) For any $Y \in D\left(A^{*}\right)$, the equality

$$
\left(Y, X_{t}\right)=\left(Y, X_{0}\right)+\int_{0}^{t}\left(-A^{*} Y, X_{s}\right) d s+\int_{0}^{t}\left\langle G^{*}\left(X_{s}\right) Y, d B_{s}\right\rangle, \quad \text { a.e. }
$$

holds for any $t \geqq 0$.

Definition 2.2 (e-solution). An evolutional solution of (2.4) is a $\mathscr{B}_{t^{-}}$ adapted process on $K$ which satisfies the condition (i) in Definition 2.1 and satisfies the following equation

$$
X_{t}=T_{t} X_{0}+\int_{0}^{t} T_{t-s} G\left(X_{s}\right) d B_{s} .
$$

The equivalence of the above two definitions of solutions is not valid in general, but the following theorems can be proved.

Theorem 2.1 (Y. Miyahara [20]). An e-solution of (2.4) is a w-solution of (2.4).

Theorem 2.2 (Y. Miyahara [19]). Suppose that $A$ has point spectrums and that $A X$ is expanded in such a form as

$$
A X=\sum \lambda_{n}\left(X, e_{n}\right) e_{n},
$$

where $\left\{e_{n}\right\}, n=1,2, \cdots$, is an orthonormal base of $K$ consisting of eigenvectors of $A$. Then a w-solution of (2.4) is an e-solution of (2.4).

The existence and the uniqueness of the solution of (2.4) is given in the form of the following theorem.

Theorem 2.3 (Y. Miyahara [20]). If $G: K \rightarrow \sigma_{2}(H, K)$ satisfies the Lipschitz condition, then the equation (2.4) has a unique e-solution.

Remark 2.1. These theorems are proved in the case of $K=H$ in [19] 
and [20]. The methods used there can be applied in the case of $K \neq H$ without any difficulties.

The equation (2.3) is considered as an equation obtained from a differential equation $d X(t) / d t=-A X(t)$ by random perturbation. From this point of view, we can treat the stability problem of the equation (2.3), and indeed they are treated in [20] (the main result is Theorem 4.2 of [20]).

\section{§3. Infinite dimensional Ornstein-Uhlenbeck process and Fokker- Planck equation}

We are interested in an equation formally described as

$$
\frac{\partial X(t, \sigma)}{\partial t}=-\hat{\omega} X(t, \sigma)+\chi(t, \sigma), \quad t \geqq 0, \quad \sigma \in[0, \pi],
$$

where $\hat{\omega}=\sqrt{-\Delta}=\sqrt{-\left(d^{2} / d \sigma^{2}\right)}$ and $\chi(t, \sigma)$ is a 2-parameter white noise. The precise definition of which will be given later.

In order to give a realization of this formal equation $(*)$ as a stochastic differential equation on a Hilbert space, we will proceed in the following manner.

Put $H=L^{2}([0, \pi])$. The Laplacian $\Delta=d^{2} / d \sigma^{2}$ on $H$ with the Neumann boundary condition is well defined; indeed $\Delta$ is a non-positive self-adjoint operator for which $\left\{-j^{2} ; j=0,1,2, \cdots\right\}$ and $\left\{\xi_{0}=1 / \sqrt{\pi}, \xi_{j}=\sqrt{2 / \pi} \cos j \sigma ; j=\right.$ $1,2, \cdots\}$ form the eigensystem. We then define an operator $\hat{\omega}$ by $\hat{\omega}=\sqrt{-\Delta}$. It is a non-negative self-adjoint operator on $H$, and $\{j\}$ and $\left\{\xi_{j}\right\}, j=0,1$, $2, \cdots$, form the eigensystem of $\hat{\omega}$.

Now we can regard the stochastic differential equation

$$
d X_{t}=-\hat{\omega} X_{t} d t+d B_{\iota}
$$

as a realization of the formal equation $(*)$, where $B_{t}$ is the c.B.m. on $H$ given by $B_{t}(\xi)=\left\langle\xi \otimes \chi_{[0, t]}, \omega\right\rangle($ see $\S 1)$.

It is easy to see that any solution to the equation (3.1) is not found in $H$ (see, e.g., Y. Miyahara [19]), so we must extend the Hilbert space $H$ to a larger Hilbert space $K$ in which the solution lives.

Since the constant part of $X_{t}$ (i.e., $\left.\left(X_{t}, \xi_{0}\right) \xi_{0}\right)$ and the remaining part are treated separately, we focus our attention on a subspace $\tilde{H}=\{\tilde{X} \in H$; $\left.\left(\tilde{X}, \xi_{0}\right)=0\right\}$ of $H$. The operator $\hat{\omega}$ is strictly positive on $\tilde{H}$ and $\hat{\omega}^{-1}$ is of Hilbert-Schmidt type, so we obtain a Hilbert scale derived from $\hat{\omega}$

$$
\tilde{H}_{\infty} \subset \cdots \subset \tilde{H}_{\alpha} \subset \cdots \subset \tilde{H}_{1} \subset \tilde{H} \subset \tilde{H}_{-1} \subset \cdots \subset \tilde{H}_{-\alpha} \subset \cdots \subset \tilde{H}_{-\infty}
$$


where $\tilde{H}_{\alpha}(0<\alpha<\infty)$ is a Hilbert space with the inner product $\left(\xi, \xi^{\prime}\right)_{\alpha}=$ $\left(\hat{\omega}^{\alpha} \xi, \hat{\omega}^{\alpha} \xi^{\prime}\right)_{\tilde{H}}$, and $\tilde{H}_{-\alpha}$ is the dual space of $\tilde{H}_{\alpha}$ (see Yu. L. Daletskii [7] for details).

We are now ready to rephrase (3.1) so as to be an equation on the extended space $\tilde{H}_{-\alpha}$,

$$
d \tilde{X}_{t}=-\hat{\omega} \tilde{X}_{t} d t+I_{0,-\alpha} d B_{t},
$$

where $I_{0,-\alpha}$ is the injection of $\tilde{H}$ into $\tilde{H}_{-\alpha}$. If $\alpha>1 / 2$, then $I_{0,-\alpha} \in \sigma_{2}\left(\tilde{H}, \tilde{H}_{-\alpha}\right)$ and we can apply the results in $\S 2$ to $\left(3.1^{\prime}\right)$ to see that $\left(3.1^{\prime}\right)$ has a unique solution. To fix the idea, we take $\alpha$ to be 1 and we have

$$
d \tilde{X}_{t}=-\hat{\omega} \tilde{X}_{t} d t+I_{0,-1} d B_{t} .
$$

As was seen in $\S 2$, the unique solution of (3.2) is given by

$$
\tilde{X}_{t}=T_{t} \tilde{X}_{0}+\int_{0}^{t} T_{t-s} d B_{s},
$$

which is called an infinite dimensional Ornstein-Uhlenbeck process.

We use the notation $\langle\xi, \tilde{X}\rangle$ to denote the dual bilinear form on $\tilde{H}_{\alpha} \times$ $\tilde{H}_{-\alpha}$, i.e. $\langle\xi, \tilde{X}\rangle=\left(\hat{\omega}^{\alpha} \xi, \hat{\omega}^{-\alpha} \tilde{X}\right)_{\tilde{H}}$ for $\xi \in \tilde{H}_{\alpha}$ and $\tilde{X} \in \tilde{H}_{-\alpha}$.

Recall that a solution to a stochastic differential equation is often made to be stationary with a suitable choice of initial probability distribution at $t=0$. This distribution is called the invariant measure.

THEOREM 3.1. The process $\tilde{X}_{t}$ has a unique invariant probability measure $\nu$, supported by $\tilde{H}_{-1 / 2}$, with the characteristic functional

$$
\begin{aligned}
c_{\nu}(\xi) & =\int_{\tilde{H}_{-1 / 2}} e^{i\langle\xi, \tilde{X}\rangle} d \nu(\tilde{X}) \\
& =\exp \left\{-\frac{1}{4}\|\xi\|_{-1 / 2}^{2}\right\}=\exp \left\{-\frac{1}{4}\left\|\hat{\omega}^{-1 / 2} \xi\right\|^{2}\right\}, \quad \xi \in \tilde{H}_{1 / 2} .
\end{aligned}
$$

Proof. By definition, the solution $X_{t}$ satisfies the following equation:

$$
\begin{aligned}
d\left\langle\xi_{j}, \tilde{X}_{t}\right\rangle & =-\left\langle\hat{\omega} \xi_{j}, \tilde{X}_{t}\right\rangle d t+d B_{t}\left(\xi_{j}\right) \\
& =-j\left\langle\xi_{j}, \tilde{X}_{t}\right\rangle d t+d B_{t}\left(\xi_{t}\right), \quad j=1,2, \cdots .
\end{aligned}
$$

Since $\left\{B_{t}\left(\xi_{j}\right)\right\}, j=1,2, \cdots$, are mutually independent 1-dimensional Brownian motions, $\left\{\left\langle\xi_{j}, \tilde{X}_{t}\right\rangle\right\}, j=1,2, \cdots$, are mutually independent 1dimensional Ornstein-Uhlenbeck processes. Therefore each $\left\langle\xi_{j}, \tilde{X}_{t}\right\rangle$ has the invariant Gaussian measure $\nu_{j}$ with mean 0 and variance $1 / 2 j$.

Let $\nu$ be the measure on $\tilde{H}_{-1}$ with characteristic functional (3.4), then 
the distribution of the random variable $\left\langle\xi_{j}, \tilde{X}\right\rangle$ is equal to $\nu_{j}$, which proves that $\nu$ is an invariant measure of $\tilde{X}_{t}$. The uniqueness of the invariant probability measure follows from that of $\nu_{j}$.

Finally we must prove that the measure $\nu$ is supported by $\tilde{H}_{-1 / 2}$. Consider a functional $C(\eta)$ on $\tilde{H}_{-1 / 2}$ given by

$$
C(\eta)=\exp \left\{-\frac{1}{4}\left\|\hat{\omega}^{-1} \eta\right\|_{-1 / 2}^{2}\right\}, \quad \eta \in \tilde{H}_{-1 / 2} .
$$

Since $\hat{\omega}^{-1}$ is a Hilbert-Schmidt operator, there exists a Gaussian measure $\tilde{\nu}$ on $\tilde{H}_{-1 / 2}$ such that

$$
\int_{\tilde{H}-1 / 2} e^{i(\eta, \tilde{X})-1 / 2} d \tilde{\nu}(\tilde{X})=C(\eta) .
$$

Put $\eta=\hat{\omega} \xi$ for $\xi \in \tilde{H}_{1 / 2}$, then $(\eta, \tilde{X})_{-1 / 2}=\left(\hat{\omega}^{1 / 2} \xi, \hat{\omega}^{-1 / 2} \tilde{X}\right)=\langle\xi, X\rangle$. We therefore have

$$
\begin{aligned}
\int_{\tilde{H}_{-1 / 2}} e^{i\langle\tilde{\xi}, \tilde{X}\rangle} d \tilde{\nu}(\tilde{X}) & =\int_{\tilde{H}_{-1 / 2}} e^{i(\eta, \tilde{X})-1 / 2} d \tilde{\nu}(\tilde{X}) \\
& =\exp \left\{-\frac{1}{4}\left\|\hat{\omega}^{-1} \eta\right\|_{-1 / 2}^{2}\right\}=\exp \left\{-\frac{1}{4}\|\xi\|_{-1 / 2}^{2}\right\} .
\end{aligned}
$$

This implies that $\nu=\tilde{\nu}$.

(Q.E.D.)

Corollary 3.1. (i) The invariant measure $\nu$ is an $\tilde{H}_{1 / 2}$-standard Gaussian measure supported by $\tilde{H}_{-1 / 2}$.

(ii) The space $\tilde{H}_{1 / 2}$ is equal to the set of all $\xi \in \tilde{H}_{-1 / 2}$ such that $\nu$ is quasi-invariant under the translation by $\xi$.

Proof. Consider a Gelfand triple

$$
\tilde{H}_{3 / 2} \subset \tilde{H}_{1 / 2} \subset \tilde{H}_{-1 / 2}
$$

where the space $\tilde{H}_{-1 / 2}^{*}$ (the dual space of $\tilde{H}_{-1 / 2}$ ) is identified with $\tilde{H}_{3 / 2}$ under the isomorphism $\tilde{H}_{1 / 2}^{*} \cong \tilde{H}_{1 / 2}$. The canonical bilinear form $\langle\langle\xi, \tilde{X}\rangle$ is expressible as

$$
\left\langle\langle\xi, \tilde{X}\rangle=\left(\hat{\omega} \xi, \hat{\omega}^{-1} \tilde{X}\right)_{1 / 2}=\left(\hat{\omega}^{3 / 2} \xi, \hat{\omega}^{-1 / 2} \tilde{X}\right)=\langle\hat{\omega} \xi, \tilde{X}\rangle .\right.
$$

Thus it holds that

$$
\begin{aligned}
\int_{\tilde{H}_{-1 / 2}} e^{i\langle\xi, \tilde{X} \|} d \nu(\tilde{X}) & =\int_{\tilde{H}_{-1 / 2}} e^{i\langle\Delta \xi, \tilde{X}\rangle} d \nu(\tilde{X}) \\
& =\exp \left\{-\frac{1}{4}\|\hat{\omega} \xi\|_{-1 / 2}^{2}\right\}=\exp \left\{-\frac{1}{4}\|\xi\|_{1 / 2}^{2}\right\}
\end{aligned}
$$


The last formula proves the assertion (i). The assertion (ii) follows from (i) by using Corollary 5.3.2 of H. Xia [25].

Theorem 3.1 assures that the equation (3.2) determines a stationary process on $\tilde{H}_{-1 / 2}$. In fact, a process $Y_{t}$ given by $Y_{t}=\int_{-\infty}^{t} T_{t-s} d B_{s}$ is a realization of such a process.

We now turn to the investigation of the continuity of $X_{t}$. The key theorem of our approach is the following (see P. Billingsley [3] or K. R. Parthasarathy [21]).

THEOREM (Kolmogorov-Prokhorov). If there are two constants $\alpha>0$ and $\beta>1$ such that

$$
E\left[\left\|\tilde{X}_{t}-\tilde{X}_{s}\right\|^{\alpha}\right] \leqq c|t-s|^{\beta}, \quad \text { for } 0 \leqq s<t \leqq T,
$$

then $\tilde{X}_{t}$ is continuous on $[0, T]$ with probability 1 .

Our result is

THEOREM 3.2. The solution of (3.2) is continuous with respect to $\tilde{H}_{-1 / 2}$ norm with probability 1.

Proof. First we assume $\tilde{X}_{0}=0$, that is $\tilde{X}_{t}=\int_{0}^{t} T_{t-s} d B_{s}$, and for notational convenience we put $Y=\tilde{X}_{t}-\tilde{X}_{s}, 0 \leqq s<t$. In order to apply the Kolmogorov-Prokhorov theorem to $\tilde{X}_{t}$ with $\alpha=4$, we will calculate $E\left[\|Y\|_{-1 / 2}^{4}\right]$.

Put $\eta_{j}=\sqrt{j} \xi_{j}, j=1,2, \cdots$. Then $\left\{\eta_{j}, j=1,2, \cdots\right\}$ is an orthonormal base in $\tilde{H}_{-1 / 2}$. Hence

$$
\begin{aligned}
{\left[E\|Y\|_{-1 / 2}^{4}\right] } & =E\left[\left(\sum_{n}\left(Y, \eta_{n}\right)_{-1 / 2}^{2}\right)^{2}\right] \\
& =\sum_{n} E\left[\left(Y, \eta_{n}\right)_{-1 / 2}^{4}\right]+\sum_{n \neq m} E\left[\left(Y, \eta_{n}\right)_{-1 / 2}^{2}\right] E\left[\left(Y, \eta_{m}\right)_{-1 / 2}^{2}\right]
\end{aligned}
$$

where it is noted that $\left(Y, \eta_{n}\right)_{-1 / 2}$ and $\left(Y, \eta_{m}\right)_{-1 / 2}$ are mutually independent if $n \neq m$. The random variable $\left(Y, \eta_{n}\right)_{-1 / 2}$ is a Gaussian random variable with mean 0 . Its variance is calculated as follows

$$
\begin{aligned}
E\left[\left(Y, \eta_{n}\right)_{-1 / 2}^{2}\right] & =E\left[\left(\left\langle\int_{0}^{t} T_{t-u}^{*} \eta_{n}, d B_{u}\right\rangle-\left\langle\int_{0}^{s} T_{s-u}^{*} \eta_{n}, d B_{u}\right\rangle\right)^{2}\right] \\
& =\frac{1}{2} n^{-2}\left\{2\left(1-e^{-n(t-s)}\right)-e^{-2 n s}\left(1-e^{-n(t-s)}\right)^{2}\right\} \\
& \leqq n^{-2}\left(1-e^{-n(t-s)}\right) .
\end{aligned}
$$

By using 


$$
E\left[\left(Y, \eta_{n}\right)_{-1 / 2}^{4}\right]=3 E\left[\left(Y, \eta_{n}\right)_{-1 / 2}^{2}\right]^{2} \leqq 3 n^{-4}\left(1-e^{-n(t-s)}\right)^{2},
$$

we have

$$
E\left[\|Y\|_{-1 / 2}^{4}\right] \leqq 3 \sum_{n} n^{-4}\left(1-e^{-n(t-s)}\right)^{2}+\left\{\sum_{n} n^{-2}\left(1-e^{-n(t-s)}\right)\right\}^{2} .
$$

We will prove that there exists a constant $\delta>1$ such that

$$
\begin{aligned}
\sum_{n} n^{-4}\left(1-e^{-n(t-s)}\right)^{2} & \leqq \text { const. }|t-s|^{\delta} \\
\sum_{n} n^{-2}\left(1-e^{-n(t-s)}\right) & \leqq \text { const. }|t-s|^{\delta / 2} .
\end{aligned}
$$

The inequality (3.6.1) follows from (3.6.2). Therefore we have only to prove (3.6.2).

Put $\gamma=t-s(>0)$ and $f(z)=z^{-2}\left(1-e^{-r^{z}}\right), z>0$. Then $f(z)>0, f^{\prime}(z)$ $<0$, and so $f(z)$ is a decreasing function. Therefore we get

$$
\sum_{n=1}^{\infty} f(n) \leqq f(1)+\int_{1}^{\infty} f(z) d z=\left(1-e^{-r}\right)+\int_{1}^{\infty} f(z) d z
$$

It is easy to see that

$$
\int_{1}^{\infty} f(z) d z=\left(1-e^{-r}\right)+\gamma \int_{r}^{\infty} \frac{e^{-v}}{v} d v
$$

and to see that

$$
\begin{gathered}
\text { if } \gamma \geqq 1, \int_{1}^{\infty} f(z) d z \leqq \text { const. } \gamma \\
\text { if } 0<\gamma<1, \int_{1}^{\infty} f(z) d z \leqq\left(1-e^{-\gamma}\right)+\gamma \int_{\gamma}^{1} \frac{e^{-v}}{v} d v+\text { const. } \gamma \\
\leqq c(\varepsilon) \gamma^{\varepsilon}, \text { for arbitrary } \varepsilon, 0<\varepsilon<1 .
\end{gathered}
$$

From (3.7), (3.8) and (3.9) it follows that

$$
\sum_{n} n^{-2}\left(1-e^{-n(t-s)}\right)=\sum_{n} f(n) \leqq c|t-s|^{\varepsilon}, \quad 0 \leqq s<t \leqq T(<\infty) .
$$

If we fix an $\varepsilon, 1 / 2<\varepsilon<1$, and put $\delta=2 \varepsilon$, then the inequality (3.10) turns into (3.6.2). Thus we have proved the theorem under the assumption $\tilde{X}_{0}$ $=0$.

In the general case where $\tilde{X}_{0} \neq 0$, the continuity of $\tilde{X}=T_{t} \tilde{X}_{0}+\int_{0}^{t} T_{t-s} d B_{s}$ follows from that of $T_{t} \tilde{X}_{0}$, because the second term has just been proved to be continuous.

(Q.E.D.) 
Since the equation (3.2) has a unique solution for any initial data $\tilde{X}_{0}$ $\in \tilde{H}_{-1 / 2}$, it determines a Markov process on $\tilde{H}_{-1 / 2}$. Let $P(t, \tilde{X}, d \tilde{Y})$ be the transition probability measure of this process. Then we obtain the following theorem.

THEOREM 3.3. The transition probability measure $P(t, \tilde{X}, d \tilde{Y})$ is equivalent to $\nu(d \tilde{Y})$ if $t>0$ and $\tilde{X} \in \tilde{H}_{-1 / 2}$.

Proof. Since the solution $\tilde{X}_{t}$ of (3.2) with initial value $\tilde{X}$ is given by $\tilde{X}_{t}=T_{t} \tilde{X}+\int_{0}^{t} T_{t-s} d B_{s}$, the transition probability measure $P(t, \tilde{X}, d \tilde{Y})$ is a Gaussian measure with mean $T_{t} \tilde{X}$ and supported by $\tilde{H}_{-1 / 2}$. The variance of $\left(\eta_{j}, \tilde{X}_{t}\right)_{-1 / 2}$ is equal to

$$
E\left[\left(\eta_{j}, \tilde{X}_{t}\right)_{-1 / 2}^{2}\right]=\frac{1}{2} j^{2}\left(1-e^{-2 j t}\right) .
$$

Therefore the covariance operator $V(t, \tilde{X})$ of $P(t, \tilde{X}, d \tilde{Y})$ is given by

$$
V(t, \tilde{X})=\frac{1}{2}\left(\hat{\omega}^{-2}-T_{t}^{2} \hat{\omega}^{-2}\right)=\frac{1}{2} \hat{\omega}^{-1}\left(I-T_{t}^{2}\right) \hat{\omega}^{-1} .
$$

While, as was seen from the proof of Theorem 3.1, the covariance operator $V_{\nu}$ of the measure $\nu$ is of the form

$$
V_{\nu}=\frac{1}{2} \hat{\omega}^{-2}
$$

These two operators are linked by $V(t, \tilde{X})=\sqrt{V_{\nu}}\left(I-T_{t}^{2}\right) \sqrt{V_{\nu}}$. Since $T_{t} \tilde{X}$ $\in \tilde{H}_{+\infty}=\bigcap_{\alpha>0} \tilde{H}_{\alpha}$ for $t>0$ and $V_{\nu}\left(\tilde{H}_{-1 / 2}\right)=\hat{\omega}^{-2}\left(\tilde{H}_{-1 / 2}\right)=\tilde{H}_{3 / 2}$, it holds that $T_{t} \tilde{X} \in V_{\nu}\left(\tilde{H}_{-1 / 2}\right)$. Hence, we can apply the well-known theorem on Gaussian measures to $\nu(d \tilde{Y})$ and $P(t, \tilde{X}, d \tilde{Y})$ (see H. Kuo [17] or A. V. Skorohod [24]) to obtain that $P(t, \tilde{X}, d \tilde{Y})$ is equivalent to $\nu(d \tilde{Y})$.

Corollary 3.2. Let $P(t, \tilde{X}, \tilde{Y})$ be the Radon-Nikodym derivative of $P(t, \tilde{X}, d \tilde{Y})$ with respect to $\nu(d \tilde{Y})$. Then $P(t, \tilde{X}, \tilde{Y})$ is expressed in the form

$$
\begin{aligned}
& P(t, \tilde{X}, \tilde{Y}) \\
& \quad=\prod_{j=1}^{\infty}\left(1-e^{-2 j t}\right)^{-1 / 2} \exp \left\{-\left(j\left(x_{j}^{2}+y_{j}^{2}\right) e^{-2 j t}-2 j x_{j} y_{j} e^{-j t}\right) /\left(1-e^{-2 j t}\right)\right\}
\end{aligned}
$$

where $\tilde{X}=\sum x_{j} \xi_{j}$ and $\tilde{Y}=\sum y_{j} \xi_{j}$.

Proof. In case $\tilde{X}=0$, the formula (3.13) is immediately obtained by the use of Theorem 3.3 of H. Kuo [17] p. 123 or Theorem 4 of A. V. Skorohod 
[24] p. 95 (note that $\sqrt{V_{\nu}}=(1 / \sqrt{2}) \hat{\omega}^{-1}$ is a Hilbert-Schmidt operator). In the general case, applying Theorem 2 of A.V. Skorohod [17] p. 83 to $P(t, \tilde{X}, d \tilde{Y})$ and $P(t, 0, d \tilde{Y})$, we get

$$
\begin{aligned}
\frac{d P(t, \tilde{X}, \cdot)}{d P(t, 0, \cdot)} & =\exp \left\{\left(V^{-1}(t, \tilde{X}) T_{t} \tilde{X}, \tilde{Y}\right)_{-1 / 2}-\frac{1}{2}\left(V^{-1}(t, \tilde{X}) T_{t} \tilde{X}, T_{t} \tilde{X}\right)_{-1 / 2}\right\} \\
& =\prod_{j} \exp \left\{\left(2 j e^{-j t} x_{j} y_{j}-j e^{-2 j t} x_{j}^{2}\right) /\left(1-e^{-2 j t}\right)\right\} .
\end{aligned}
$$

The formula (3.13) follows from this by the use of the formula

$$
P(t, \tilde{X}, \tilde{Y})=\frac{d P(t, \tilde{X}, .)}{d \nu(\cdot)}=\frac{d P(t, \tilde{X}, \cdot)}{d P(t, 0, \cdot)} \times \frac{d P(t, 0, \cdot)}{d \nu(\cdot)} .
$$

With the explicit expression of $P(t, \tilde{X}, \tilde{Y})$ we are able to speak of its further properties.

TheOREM 3.4. The function $P(t, \tilde{X}, \tilde{Y})$ has the following properties:

(i) $P(t, \tilde{X}, \tilde{Y})$ is a continuous function on $(0, \infty) \times \tilde{H}_{-1 / 2} \times \tilde{H}_{-1 / 2}$.

(ii) $P(t, \tilde{X}, \tilde{Y})=P(t, \tilde{Y}, \tilde{X})$, that is $P(t, \tilde{X}, \tilde{Y})$ is symmetric.

(iii) For fixed $t>0$ and $\tilde{X} \in \tilde{H}_{-1 / 2}, P(t, \tilde{X}, \tilde{Y})$ is an $L^{p}(d \nu)$-function of $\tilde{Y}$ for every $p>0$. But $P(t, \tilde{X}, \tilde{Y})$ is not bounded.

(iv) $P(t, \tilde{X}, \tilde{Y})$ is the fundamental solution of the following equation

$$
\begin{gathered}
\frac{\partial P(t, \tilde{X}, \tilde{Y})}{\partial t}=\frac{1}{2} \Delta_{V} P(t, \tilde{X}, \tilde{Y})-\int_{0}^{\pi} \hat{\omega} \tilde{Y}(\sigma) \frac{\delta P(t, \tilde{X}, \tilde{Y})}{\delta(\tilde{Y} \sigma)} d \sigma, \\
P(t, \tilde{X}, \tilde{Y}) \longrightarrow \delta_{\tilde{X}}(\tilde{Y}) \quad(t \downarrow 0),
\end{gathered}
$$

where $\Delta_{V}$ denotes the Volierra Laplacian and $\delta P / \delta \tilde{Y}(\sigma)$ denotes the functional derivative (see I. Kubo [16] $\S 7$ and Remark 3.1 given below), and where the precise meaning of (3.15) is that

$$
\int_{\tilde{H}_{-1 / 2}} P(t, \tilde{X}, \tilde{Y}) f(\tilde{Y}) d \nu(\tilde{Y}) \longrightarrow f(\tilde{X}) \quad \text { as } t \downarrow 0
$$

for any continuous bounded function $f(\tilde{Y})$ of $\tilde{H}_{-1 / 2}$.

Before we come to the proof we need some interpretation of the Volterra Laplacian and a lemma.

Remark 3.1. The function $P(t, \tilde{X}, \tilde{Y})$ can naturally be extended to be a continuous function on $(0, \infty) \times \tilde{H}_{-1} \times \tilde{H}_{-1}$, and then $P(t, \tilde{X}, \tilde{Y})$ can be expanded in the same form as (3.13) on the extended domain $(0, \infty) \times \tilde{H}_{-1}$ $\times \tilde{H}_{-1}$. Putting $E=\tilde{H}_{1}$ and $E^{*}=\tilde{H}_{-1}$ in I. Kubo's notations, we know 
that $\Delta_{V}$ is well-defined in the sense of $\S 7$ of I. Kubo [16]. The functional derivative $\delta P / \delta \tilde{Y}(\sigma)$ can be understood to be an element of $\tilde{H}_{-2}^{*}$ in the same way. Since $\hat{\omega} Y \in \tilde{H}_{-2}$, the dual product $\int \hat{\omega} \tilde{Y}(\sigma)(\delta P / \delta \tilde{Y}(\sigma)) d \sigma$ is well-defined. In our cases, the Volterra Laplacian $\Delta_{V} P$ can be expressed in the form

$$
\Delta_{V} P(t, \tilde{X}, \tilde{Y}) \doteq \int_{0}^{\pi} P^{(2)}(t, \tilde{X}, \tilde{Y} ; \sigma, \sigma) d \sigma
$$

where $P^{(2)}\left(t, \tilde{X}, \tilde{Y} ; \sigma, \sigma^{\prime}\right)$ is the second functional derivative of $P(t, \tilde{X}, \tilde{Y})$.

Lemma 3.1. Let $\left\{X_{n}\right\}, n=1,2, \cdots$, be a sequence of random variables such that $X_{n} \rightarrow X$ a.e. and $\sup _{n} E\left[\left|X_{n}\right|^{p}\right]<\infty$. Then $X_{n} \rightarrow X$ in $L^{x^{\prime}}$ for all $p^{\prime}, 0<p^{\prime}<p$.

We are now ready to give a proof of the Theorem 3.4.

Proof of Theorem 3.4. Put

$$
\left.\phi_{J}(t, x, y)=\left(1-e^{-2 \jmath t}\right)^{-1 / 2} \exp \left\{j\left(x^{2}+y^{2}\right) e^{-2 j t}-2 j x y e^{-j t}\right) /\left(1-e^{-2 j t}\right)\right\}
$$

and

$$
P_{n}(t, \tilde{X}, \tilde{Y})=\prod_{j=1}^{n} \phi_{j}\left(t, x_{j}, y_{j}\right), \quad \tilde{X}=\sum x_{j} \xi_{j} \text { and } \tilde{Y}=\sum y_{j} \xi_{j} .
$$

Then $P_{n}(\dot{t}, \tilde{X}, \tilde{Y}) \rightarrow P(t, \tilde{X}, \tilde{Y})$ (as $\left.n \rightarrow \infty\right)$ uniformly on $T \times A \times B \subset(0, \infty)$ $\times \tilde{H}_{-1 / 2} \times \tilde{H}_{-1 / 2}$, where $T$ is a compact subset of $(0, \infty)$ and where $A$ and $B$ are respectively bounded subsets of $\tilde{H}_{-1 / 2}$. This proves (i). The assertion (ii) is obvious from (3.13).

We then come to the proof of (iii). First, observe the moment $\int\left|P_{n}(t, \tilde{X}, \tilde{Y})\right|^{p} d \nu(\tilde{Y})$,

$$
\begin{aligned}
\int\left|P_{n}(t, \tilde{X}, \tilde{Y})\right|^{p} d \nu(\tilde{Y})=\prod_{j=1}^{n} \int_{-\infty}^{\infty}\left|\phi_{j}\left(t, x_{j}, y\right)\right|^{p} d \nu_{j}(y) \\
=\prod_{j=1}^{n}\left[\left(1-e^{-2 j t}\right)^{-(p-1) / 2}\left(1+(p-1) e^{-2 j t}\right)^{-1 / 2}\right. \\
\left.\quad \times \exp \left\{\left[-j p\left(1-p+(p-1) e^{-2 j t}\right) e^{-2 j t} x_{j}^{2}\right] /\left[\left(1-e^{-2 j t}\right)\left(1+(p-1) e^{-2 j t}\right)\right]\right\}\right] .
\end{aligned}
$$

It is easy to see that this product converges (as $n \rightarrow \infty$ ) if $\sum x_{j}^{2} / j^{\alpha}<\infty$ for some $\alpha>0$, and in fact this condition is always satisfied for $\tilde{X} \in \tilde{H}_{-1 / 2}$. Therefore $\left\{\int\left|P_{n}\right|^{P} d \nu(\tilde{Y}), n=1,2, \cdots,\right\}$ is bounded. Applying Lemma 3.1 to $\left\{P_{n}\right\}, n=1,2, \cdots$, and $P$, we prove that $P(t, \tilde{X}, \cdot) \in L^{P}$.

Let $\tilde{X}$ be an element of $\tilde{H}_{-1 / 2}$ such that $\sum j x_{j}^{2}=\infty$. The maximum 
of $P_{n}(t, \tilde{X}, \tilde{Y})$ is $\prod_{j=1}^{n}\left(1-e^{-2 j t}\right)^{-1 / 2} \exp \left\{i x_{j}^{2}\right\}$ which is attained by $y_{j}=e^{j t} x_{j}$, $j=1, \cdots, n$. Put $\tilde{Y}_{n}=\sum_{j=1}^{n} e^{j t} x_{j} \xi_{j}$. Then the sequence

$$
P\left(t, \tilde{X}, \tilde{Y}_{n}\right)=\prod_{j=1}^{n}\left(1-e^{-2 j t}\right)^{-1 / 2} \exp \left\{j x_{j}^{2}\right\} \prod_{j=n+1}^{\infty}\left(1-e^{-2 j t}\right), \quad n=1,2, \cdots,
$$

diverges as $n \rightarrow \infty$. Thus we have proved the unboundedness of $P(t, \tilde{X}, \tilde{Y})$.

Finally we will prove the assertion (iv). It is easy to see that the function $\sum_{j=1}^{n} z_{j}\left(\partial \phi_{j}\left(t, x_{j}, y_{j}\right) / \partial y_{j}\right)$ of $\tilde{Z}$ and $\tilde{Y}$ (where $t>0$ and $\tilde{X} \in H_{-1 / 2}$ are fixed) converges uniformly on every bounded set $B \times B^{\prime} \subset \tilde{H}_{-1 / 2} \times \tilde{H}_{-1 / 2}$. From this it follows that $P(t, \tilde{X}, \tilde{Y})$ is Fréchet-differentiable as a function of $\tilde{Y}$. Similarly, it is easily verified that $P(t, \tilde{X}, \tilde{Y})$ is $k$-times Fréchetdifferentiable, where $k$ may be taken arbitrarily large.

Using the equation

$$
\frac{\partial \phi_{j}}{\partial t}=\frac{1}{2} \frac{\partial \phi_{j}}{\partial y^{2}}-j y \frac{\partial \phi_{j}}{\partial y}
$$

we get

$$
\frac{\partial \phi_{j}}{\partial t}(t, x, y)=\phi_{j}(t, x, y) \times \psi_{j}(t, x, y)
$$

where

$$
\begin{aligned}
\psi_{j}(t, x, y)= & 2 j^{2} e^{-2 j t}\left(y e^{-j t}-x\right)^{2} /\left(1-e^{-2 j t}\right)^{2}-j e^{-2 j t} /\left(1-e^{-2 j t}\right) \\
& +2 j^{2} e^{-j t}\left(y^{2} e^{-j t}-x y\right) /\left(1-e^{-2 j t}\right) .
\end{aligned}
$$

Therefore we obtain

$$
\begin{aligned}
\frac{\partial P_{n}(t, \tilde{X}, \tilde{Y})}{\partial t} & =\sum_{j=1}^{n}\left\{\frac{\partial \phi_{j}\left(t, x_{j}, y_{j}\right)}{\partial t} \prod_{k \neq j}^{n} \phi_{k}\left(t, x_{k}, y_{k}\right)\right\} \\
& =\left\{\sum_{j=1}^{n} \psi_{j}\left(t, x_{j}, y_{j}\right)\right\} P_{n}(t, \tilde{X}, \tilde{Y}) .
\end{aligned}
$$

It is clear that the sequences $\left\{\sum_{j=1}^{n} \psi_{j}\right\}$ and $\left\{P_{n}\right\}$ converge uniformly on any $t$-interval $(a, b), 0<a<b<\infty$. We therefore prove that there exists the derivative $\partial P / \partial t$ and it satisfies

$$
\frac{\partial P}{\partial t}=\lim _{n \rightarrow \infty} \frac{\partial P_{n}}{\partial t}=\left(\sum_{j=1}^{\infty} \psi_{j}\right) P(t, \tilde{X}, \tilde{Y}) .
$$

The functional derivatives of $P$ is calculated as follows

$$
P^{\prime}\left(t, \tilde{X}, \tilde{Y} ; \xi_{j}\right)=\left\{2 j\left(x_{j} e^{-j t}-y_{j} e^{-2 j t}\right) /\left(1-e^{-2 j t}\right)\right\} P(t, \tilde{X}, \tilde{Y}),
$$




$$
\begin{aligned}
P^{(2)}\left(t, X, Y ; \xi_{j}, \xi_{j}\right)= & \left\{\left[2 j\left(x_{j} e^{-j t}-y_{j} e^{-2 j t}\right) /\left(1-e^{-2 j t}\right)\right]^{2}\right. \\
& \left.-2 j e^{-2 j t} /\left(1-e^{-2 j t}\right)\right\} P(t, \tilde{X}, \tilde{Y}) .
\end{aligned}
$$

Thus we have

$$
\begin{aligned}
& \frac{1}{2} \Delta_{V} P(t, \tilde{X}, \tilde{Y}) \\
& \quad=\left[\sum_{j=1}^{\infty}\left\{2 j^{2} e^{-2 j t}\left(x_{j}-y_{j} e^{-j t}\right)^{2} /\left(1-e^{-2 j t}\right)^{2}-j e^{-2 j t} /\left(1-e^{-2 j t}\right)\right\}\right] P(t, \tilde{X}, \tilde{Y}) .
\end{aligned}
$$

On the other hand, it holds that

$$
\begin{aligned}
P^{\prime}(t, \tilde{X}, \tilde{Y} ; \sigma) & =P^{\prime}\left(t, \tilde{X}, \tilde{Y} ; \delta_{\sigma}\right) \\
& =\sum_{j} \xi_{j}(\sigma) P^{\prime}\left(t, \tilde{X}, \tilde{Y} ; \xi_{j}\right) \\
& =\left[\sum_{j}\left\{2 j\left(x_{j} e^{-j t}-y_{j} e^{-2 j t}\right) /\left(1-e^{-2 j t}\right)\right\} \xi_{j}(\sigma)\right] P(t, \tilde{X}, \tilde{Y}) .
\end{aligned}
$$

From the formula $\hat{\omega} \tilde{Y}(\sigma)=\sum_{j} j y_{j} \xi_{j}(\sigma)$, it follows that

$$
\begin{aligned}
& \int_{0}^{\pi} \hat{\omega} \tilde{Y}(\sigma) P^{\prime}(t, \tilde{X}, \tilde{Y} ; \sigma) d \sigma \\
& \quad=\left\{\sum_{j} 2 j^{2} y_{j}\left(x_{j} e^{-j t}-y_{j} e^{-2 t j}\right) /\left(1-e^{-2 j t}\right)\right\} P(t, \tilde{X}, \tilde{Y}) .
\end{aligned}
$$

Finally we obtain

$$
\begin{array}{rl}
\frac{1}{2} \Delta_{V} & P(t, \tilde{X}, \tilde{Y})-\int \hat{\omega} \tilde{Y}(\sigma) \frac{\delta P}{\delta \tilde{Y}(\sigma)} d \sigma \\
= & {\left[\sum_{j} \psi_{j}(t, \tilde{X}, \tilde{Y})\right] P(t, \tilde{X}, \tilde{Y}) .}
\end{array}
$$

From (3.16) and (3.17) it follows that $P(t, \tilde{X}, \tilde{Y})$ satisfies the equation (3.14). From the definition of $P(t, \tilde{X}, \tilde{Y})$, it follows that

$$
\int P(t, \tilde{X}, \tilde{Y}) f(\tilde{Y}) d \nu(\tilde{Y})=\int f(\tilde{Y}) P(t, \tilde{X}, d \tilde{Y})=E\left[f\left(\tilde{X}_{t}\right)\right], \quad \tilde{X}_{0}=\tilde{X} .
$$

Since $\tilde{X}_{t}$ is continuous on $\tilde{H}_{-1 / 2}$ and since $f(\tilde{Y})$ is a bounded continuous function on $\tilde{H}_{-1 / 2}$, we have

$$
\lim _{t \downarrow 0} E\left[f\left(\tilde{X}_{t}\right)\right]=E\left[f\left(\tilde{X}_{0}\right)\right]=f(\tilde{X}) .
$$

Thus the condition (3.15) is satisfied, which proves the theorem. (Q.E.D.)

Remark 3.2. Theorem 3.4 holds true even if the function $P(t, \tilde{X}, \tilde{Y})$ is considered as a function on $(0, \infty) \times \tilde{H}_{-\alpha} \times \tilde{H}_{-\alpha}$ for any $\alpha, 1 / 2 \leqq \alpha<\infty$.

Let $\left\{U_{t}\right\}, t \geqq 0$, be the semi-group on $L^{\infty}\left(\tilde{H}_{-1 / 2}, \nu\right)$ determined by the 
transition probability measure $P(t, \tilde{X}, d \tilde{Y})$, that is

$$
\begin{aligned}
\left(U_{t} f\right)(\tilde{X}) & =\int_{\tilde{H}_{-1 / 2}} P(t, \tilde{X}, d \tilde{Y}) f(\tilde{Y})=\int_{\tilde{H}_{-1 / 2}} P(t, \tilde{X}, \tilde{Y}) f(\tilde{Y}) d \nu(\tilde{Y}) \\
& =E_{\tilde{X}}\left[f\left(\tilde{X}_{t}\right)\right], \quad f \in L^{\infty}\left(\tilde{H}_{-1 / 2}, \nu\right),
\end{aligned}
$$

and let $L$ be the infinitesimal generator of the semi-group $\left\{U_{t}\right\}$. We denote by $C_{0}^{2}\left(\boldsymbol{R}^{k}\right)$ the class of twice continuously differentiable functions with compact support in $R^{k}$. Then we obtain the following theorem.

THEOREM 3.5. A function $F(\tilde{Y})$, given by

$$
F(\tilde{Y})=f\left(v_{1}, \cdots, v_{k}\right), \quad v_{j}=\left\langle\xi_{j}, \tilde{Y}\right\rangle, \quad j=1,2, \cdots, k, f \in C_{0}^{2}\left(\boldsymbol{R}^{k}\right),
$$

belongs to the domain of $L$ and satisfies

$$
(L F)(\tilde{Y})=\left[\frac{1}{2} \sum_{j=1}^{k} \frac{\partial^{2} f}{\partial v_{j}^{2}}-\sum_{j=1}^{k} j v_{j} \frac{\partial f}{\partial v_{j}}\right]_{v_{j}=\langle\xi \xi \tilde{Y}\rangle} \cdot
$$

Proof. We need only to prove (3.18). A process $X_{t}^{k}$ given by

$$
X_{t}^{k}=\left(x_{t}^{1}, \cdots, x_{t}^{k}\right), \quad x_{t}^{j}=\left\langle\xi_{j}, \tilde{X}_{t}\right\rangle
$$

is a $k$-dimensional Ornstein-Uhlenbeck process, and the components of $X_{t}^{k}$ satisfy

$$
d x_{t}^{j}=-j x_{t}^{j} d t+d B_{t}(\xi), \quad j=1,2, \cdots, k .
$$

Now, the conclusion of the theorem follows.

Corollary 3.2. Let $D(L)$ denote the domain of the operator $L$. Then $D(L) \cap L^{2}(d \nu)$ is dense in $L^{2}(d \nu)$,

Proof. The set

$$
\begin{aligned}
C_{0}^{2}=\left\{F \in L^{2}(d \nu) ;\right. & F(\tilde{Y})=f\left(v_{1}, \cdots, v_{k}\right), v_{j}=\left\langle\xi_{j}, \tilde{Y}\right\rangle, \\
& \left.j=1,2, \cdots, k, f \in C_{0}^{2}\left(R^{k}\right), k=1,2, \cdots\right\},
\end{aligned}
$$

is dense in $L_{2}(d \nu)$. Since $C_{0}^{2} \subset D(L)$ by Theorem 3.5, $D(L) \cap L^{2}(d \nu)$ is dense in $L^{2}(d \nu)$.

(Q.E.D).

By Corollary 3.2, $L$ can be considered as an operator on $L^{2}\left(\tilde{H}_{-1 / 2}, d \nu\right)$. Let the closed extension on $L$ be denoted by the same symbol $L$.

Noting that $\nu$ is the $\tilde{H}_{1 / 2}$-standard Gaussian measure on $\tilde{H}_{-1 / 2}$, $L^{2}\left(\tilde{H}_{-1 / 2}, d \nu\right)$ is decomposed in the form

$$
L^{2}\left(\tilde{H}_{-1 / 2}, d \nu\right)=\sum_{n=0}^{\infty} \oplus \mathscr{H}_{n} \quad \text { (Wiener's direct sum decomposition) }
$$


where $\mathscr{H}_{n}$ is the closed subspace spanned by $\left\{\prod_{j} H_{n_{j}}\left(\sqrt{j}\left\langle\xi_{j}, \tilde{Y}\right\rangle\right), \sum_{j} n_{j}=\right.$ $n\}\left(H_{n}\right.$ Hermite polynomial of degree $\left.n\right)$ (see $\S 4$ or T. Hida [14]). We note here that

$$
\begin{aligned}
\left\{\Phi_{\{n\}}=\left(\prod_{j} n_{j} ! 2^{n_{j}}\right)^{-1 / 2} \prod_{j} H_{n_{j}}\left(\sqrt{j}\left\langle\xi_{j}, \tilde{Y}\right\rangle\right), n_{j}\right. & =0,1, \cdots, \\
j & \left.=1,2, \cdots, \sum n_{j}<\infty\right\}
\end{aligned}
$$

is an orthonormal base for $L^{2}(d \nu)$.

THEOREM 3.6. (i) The functions $\left\{\Phi_{\left\{n_{j}\right\}}(\tilde{Y}), n_{j}=0,1, \cdots, \sum n_{j}<\infty\right\}$, satisfy the following formula

$$
L \Phi_{\left\{n_{j\}}\right\}}(\tilde{Y})=-\left(\sum_{j} j n_{j}\right) \Phi_{\left\{n_{j}\right\}}(\tilde{Y}) .
$$

The operator $L$, acting on $L^{2}(d \nu)$, is non-positive definite and self-adjoint.

(ii) The function $P(t, \tilde{X}, \tilde{Y})$ satisfies the equation

$$
\frac{d P}{d t}(t, \tilde{X}, \tilde{Y})=L P(t, \tilde{X}, \tilde{Y}), \quad t>0, \text { for fixed } \tilde{X} .
$$

Proof. When $F(\tilde{Y})$ is expressed in the form

$$
F(\tilde{Y})=f\left(v_{1}, \cdots, v_{k}\right), \quad v_{j}=\left\langle\xi_{j}, \tilde{Y}\right\rangle, \quad j=1, \cdots, k,
$$

with a polynomial $f$ in $v_{j}^{\text {'s, }} F(\tilde{Y})$ satisfies

$$
(L F)(\tilde{Y})=\left[\frac{1}{2} \sum_{j=1}^{k} \frac{\partial^{2} f}{\partial v_{j}^{2}}-\sum_{j=1}^{k} \frac{\partial f}{\partial v_{j}}\right]_{v_{j}=\left\langle\xi_{j}, \tilde{Y}\right\rangle} .
$$

If, in particular, $f\left(v_{1}, \cdots, v_{k}\right)=\prod_{j=1}^{k} H_{n_{j}}\left(\sqrt{j} v_{j}\right)$, then we have

$$
\begin{gathered}
L \prod_{j=1}^{k} H_{n_{j}}\left(\sqrt{j}\left\langle\xi_{j}, \tilde{Y}\right\rangle\right)=\left[\frac{1}{2} \sum \frac{\partial^{2} f}{\partial v_{j}^{2}}-\sum j v_{j} \frac{\partial f}{\partial v_{j}}\right]_{v_{j}=\langle\hat{\xi} j, \tilde{Y}\rangle} \\
=\sum_{j}\left[\left\{\frac{1}{2} j \frac{\partial^{2} H_{n_{j}}}{\partial x_{j}^{2}}-j x_{j} \frac{\partial H_{n_{j}}}{\partial x_{j}}\right\} \prod_{i \neq j} H_{n_{i}}\right]_{x_{j}=\sqrt{j}\langle\hat{j}, \tilde{Y}\rangle} .
\end{gathered}
$$

Using a formula

$$
\frac{d^{2} H_{n}}{d x^{2}}-2 x \frac{d H_{n}}{d x}+2 n H_{n}=0
$$

for the Hermite polynomial, we obtain

$$
L \prod_{j=1}^{k} H_{n_{j}}\left(\sqrt{j}\left\langle\xi_{j}, \tilde{Y}\right\rangle\right)=-\left(\sum_{j} j n_{j}\right) \prod_{i} H_{n_{i}}\left(\sqrt{i}\left\langle\xi_{i}, \tilde{Y}\right\rangle\right),
$$


which proves (3.20). Since $\left\{\Phi_{\left\{n_{j}\right\}}(\tilde{Y})\right\}$ is an orthonormal base for $L^{2}(d \nu)$, the formula (3.20) proves that $L$ is non-positive definite and self-adjoint.

Let $P_{n}(t, \tilde{X}, \tilde{Y})$ and $\psi_{k}(t, x, y)$ be functions given in the proof of Theorem 3.4. Because $P_{n}(t, \tilde{X}, \tilde{Y})$ is a tame function, it is easily seen that

$$
\frac{\partial P_{n}}{\partial t}(t, \tilde{X}, \tilde{Y})=L P_{n}(t, \tilde{X}, \tilde{Y})=\left(\sum_{k=1}^{n} \psi_{k}\left(t, x_{k}, y_{k}\right)\right) P_{n}(t, \tilde{X}, \tilde{Y}) \text {. }
$$

Recall that

$$
P_{n}(t, \tilde{X}, \tilde{Y}) \longrightarrow P(t, \tilde{X}, \tilde{Y}) \text { in } L^{2}(d \nu) \text {. }
$$

We can further prove that

$$
\frac{\partial P_{n}}{\partial t}(t, \tilde{X}, \tilde{Y}) \longrightarrow \frac{\partial P}{\partial t}(t, \tilde{X}, \tilde{Y}) \quad \text { in } L^{2}(d \nu)
$$

and

$$
L P_{n}(t, \tilde{X}, \tilde{Y})=\left(\sum_{k=1}^{n} \psi_{k}\right) P_{n}(t, \tilde{X}, \tilde{Y}) \longrightarrow\left(\sum_{k=1}^{\infty} \psi_{k}\right) P(t, \tilde{X}, \tilde{Y}) \quad \text { in } L^{2}\left(d_{\nu}\right) .
$$

Since the operator $L$ is closable, the formulae (3.24) and (3.25) prove that $P(t, \tilde{X}, \tilde{Y}) \in D(L), t>0$, and that

$$
L P(t, \tilde{X}, \tilde{Y})=\lim _{n \rightarrow \infty} L P_{n}(t, \tilde{X}, \tilde{Y})=\left(\sum_{k=1}^{\infty} \psi_{k}\right) P(t, \tilde{X}, \tilde{Y}) .
$$

It is easily verified that $d P / d t$ the derivative of $P(t, \tilde{X}, \cdot)$ in $L^{2}(d \nu)$ norm is equal to the point-wise partial derivative $\partial P / \partial t$. Therefore the equality (3.21) follows from (3.22), (3.24) and (3.26).

(Q.E.D.)

We will now consider a generalized equation of (3.2)

$$
d \tilde{X}_{t}=-\hat{\omega} \tilde{X}_{t} d t+B d B_{t},
$$

where $B$ is a bounded linear operator defined on $\tilde{H}$. We can carry on the same analysis on (3.27) as we have done on:(3.2), to obtain the following results:

1. There exists a unique invariant probability measure $\nu_{B}$ supported by $\tilde{H}_{-1 / 2}$ and its characteristic function $C_{\nu_{B}}(\xi)$ is given by

$$
C_{\nu_{B}}(\xi)=\int_{\tilde{H}_{-1 / 2}} e^{-i\langle\xi, \tilde{X}\rangle} d \nu_{B}(\tilde{X})=\exp \left\{-\frac{1}{4}\left\|B^{*} \hat{\omega}^{1 / 2} \xi\right\|^{2}\right\}, \quad \xi \in \tilde{H}_{1 / 2} .
$$

2. There is a dichotomy that the measures $\nu_{B}$ and $\nu$ are either mutually 
equivalent or singular. They are equivalent if and only if $\hat{\omega}^{1 / 2} B B^{*} \hat{\omega}^{-1 / 2}-I$ is of Hilbert-Schmidt type.

3. The solution of (3.27) is continuous in $\tilde{H}_{-1 / 2}$ norm.

4. In order that the transition probability measure $P_{B}(t, \tilde{X}, d \tilde{Y})$ is equivalent to $\nu_{B}(d \tilde{Y})$, it is necessary and sufficient that $T_{t} \tilde{X} \in S_{1}\left(\tilde{H}_{-1 / 2}\right)$ and that $S_{1}^{-1 / 2} T_{t} S_{1} T_{t} S_{1}^{-1 / 2}$ is of Hilbert-Schmidt type, where $S_{1}=(1 / 2) \hat{\omega}^{-1 / 2} B B^{*} \hat{\omega}^{-1 / 2}$.

5. A necessary and sufficient condition for $P_{B}(t, \tilde{X}, \tilde{Y})$ to be $\nu_{B}$-symmetric is that the operator $B B^{*}$ commutes with $\hat{\omega}$.

Remark 3.3. Our methods are applicable to some generalized cases. Let $H$ be any real separable Hilbert space and let $A$ be a positive unbounded operator on $H$ such that $A^{-1}$ is of Hilbert-Schmidt type. Then, for the equation on $H$ of the form

$$
d X_{t}=-A X_{t} d t+d B_{t}
$$

we can do the same discussions as we have done for the equation (3.2).

\section{$\S 4$. Multiple Wiener integrals on a Hilbert space}

In this section we will first consider the Wiener's direct sum decompositions of $L^{2}\left(\mathscr{E}^{*}, \mu\right)$ and $L^{2}\left(\mathscr{E}^{*} \rightarrow K\right)$, and then we will proceed to investigate their integral representations. (See $\S 1$ for the notations.)

1. Functionals of multi-parameter white noise.

Introduce the space of real valued functionals of white noise

$$
\begin{aligned}
\left(L^{2}\right)_{D}=L^{2}\left(\mathscr{E}^{*}\right) & =L^{2}\left(\mathscr{E}^{*}, \mathscr{B}, \mu\right) \\
& =\left\{\phi ; \int_{\delta^{*}}|\phi(\omega)|^{2} d \mu(\omega)<\infty\right\} .
\end{aligned}
$$

Then we are able to obtain the Wiener's direct sum decomposition of $\left(L^{2}\right)_{\nu}$ as follows.

For any $\phi \in\left(L^{2}\right)_{D}$ define $\mathscr{T} \phi$ by

$$
(\mathscr{T} \phi)(\eta)=\int_{\mathscr{E}^{*}} e^{i\langle\eta, \omega\rangle} \phi(\omega) d \mu(\omega), \quad \eta \in \mathscr{E} .
$$

Putting

$$
\phi(\omega)=\exp \left\{2 t \frac{\langle\tilde{\eta}, \omega\rangle}{\sqrt{2}}-t^{2}\right\}=\sum_{k} \frac{t^{k}}{k !} H_{k}(\langle\tilde{\eta}, m\rangle / \sqrt{2}), \quad\|\tilde{\eta}\|=1,
$$

where $H_{k}()$ is the Hermite polynomial of degree $k$, we obtain 


$$
(\mathscr{T} \phi)(\eta)=C_{\mu}(\eta) \sum_{k=1}^{\infty} \frac{(i \sqrt{2} t)^{k}}{k !}(\eta, \tilde{\eta})^{k}
$$

Therefore we have proved

$$
\left(\mathscr{T} H_{k}(\langle\tilde{\eta}, \omega\rangle / \sqrt{2})\right)(\eta)=C_{\mu}(\eta)(\sqrt{2} i)^{k}(\tilde{\eta}, \eta)^{k}, \quad\|\tilde{\eta}\|=1 .
$$

We then show a generalization of (4.2). Put $\phi(\omega)=\prod_{j=1}^{m} H_{K_{j}}\left(\left\langle\eta_{j}, \omega\right\rangle / \sqrt{2}\right)$, $\sum K_{j}=n$, where $\left\{\eta_{j}\right\}, \eta_{j} \in \mathscr{E}$, is an orthonormal system in $\mathscr{H}=L^{2}(D \times T)$. Then we can easily prove that

$$
\begin{aligned}
(\mathscr{T} \phi)(\eta) & =C_{\mu}(\eta)(\sqrt{2} i)^{n} \prod_{j=1}^{m}\left(\eta, \eta_{j}\right)^{k_{j}} \\
& =C_{\mu}(\eta) i^{n} \int_{(D \times T)^{n}} \cdots \int F\left(x_{1}, \cdots, x_{n}\right) \eta^{n \otimes}\left(x_{1}, \cdots, x_{n}\right) d x_{1} \cdots d x_{n} \\
& =C_{\mu}(\eta) i^{n} \int_{(D \times T)^{n}} \cdots \int \tilde{F}\left(x_{1}, \cdots, x_{n}\right) \eta^{n \otimes}\left(x_{1}, \cdots, x_{n}\right) d x_{1} \cdots d x_{n}
\end{aligned}
$$

where $F\left(x_{1}, \cdots, x_{n}\right)=2^{n / 2}\left[\eta_{1}\left(x_{1}\right) \cdots \eta_{1}\left(x_{k_{1}}\right)\right] \cdots\left[\cdots \eta_{m}\left(x_{n}\right)\right]$, and $\tilde{F}\left(x_{1}, \cdots, x_{n}\right)$ $=$ symmetrization of $F\left(x_{1}, \cdots, x_{n}\right)=1 / n ! \sum_{\pi} F\left(x_{\pi(1)}, \cdots, x_{\pi(n)}\right)$, $\pi$; permutation of $(1,2, \cdots, n)$, and $\eta^{n \otimes}=n$-times tensor product of $\eta$.

The following formulae can easily be proved:

$$
\|F\|_{L^{2}}=2^{n / 2}, \quad\|\tilde{F}\|_{L^{2}}=2^{n / 2}(n !)^{-1 / 2}\left(\prod_{j=1}^{m} k_{j} !\right)^{1 / 2} .
$$

On the other hand it is obvious that

$$
\|\phi\|_{\left(L^{2}\right) D}=\left(\prod_{j=1}^{m} k_{j} !\right)^{1 / 2} 2^{n / 2},
$$

and so we have established

$$
\|\phi\|_{\left(L^{2}\right)_{D}}=(n !)^{1 / 2}\|\tilde{F}\|_{L^{2}\left((D \times T)^{n}\right)} .
$$

Let $\mathscr{H}_{n}$ be the subspace of $\left(L^{2}\right)_{D}$ spanded by the Hermite polynomials of degree $n$. Then we can prove

$$
\left(L^{2}\right)_{D}=\sum_{n=0}^{\infty} \oplus \mathscr{H}_{n} \quad \text { (Wiener's direct sum decomposition). }
$$

In fact the correspondence between $\phi=\prod H_{k_{j}}\left(\left\langle\eta_{j}, \omega\right\rangle / \sqrt{2}\right)$ and $\tilde{F} \epsilon$ $\hat{L}^{2}\left((D \times T)^{n}\right)$ can be extended to the one-to-one mapping from $\mathscr{H}_{n}$ to $\hat{L}^{2}\left((D \times T)^{n}\right), n=0,1,2, \cdots$, where $\hat{L}^{2}\left((D \times T)^{n}\right)=\left\{F ; F \in L^{2}\left((D \times T)^{n}\right)\right.$ and $F$ is symmetric\}. We denote this transformation from $\mathscr{H}_{n}$ to $\hat{L}^{2}\left((D \times T)^{n}\right)$ by $\tau$. Thus we have obtained the following diagram. 
$\left(L^{2}\right)_{D}=\sum \oplus \mathscr{H}_{n} \cong \sum \oplus \sqrt{n !} \hat{L}^{2}\left((D \times T)^{n}\right), \quad$ under $\tau:$

$\tau: \phi \longrightarrow \tau \phi \in \hat{L}^{2}\left((D \times T)^{n}\right), \quad \phi \in \mathscr{H}_{n}$,

$(\mathscr{T} \phi)(\eta)=i^{n} C(\eta) \int_{(D \times T)^{n}} \cdots \int \tau \phi\left(x_{1}, \cdots, x_{n}\right) \eta^{n \otimes}\left(x_{1}, \cdots, x_{n}\right) d x_{1} \cdots d x_{n}$,

$\|\phi\|_{\left(L^{2}\right) D}=\sqrt{n !}\|\tau \phi\|_{L^{2}\left((D \times T)^{n}\right)}$.

Definition 4.1. $\tau \phi$ is called the kernel of the integral representation of $\phi$.

EXAMPLE 4.1.

$\tau\left(\prod_{j} H_{k_{j}}\left(\left\langle\eta_{j}, \omega\right\rangle / \sqrt{2}\right)\right)=$ the symmetrization of $2^{n / 2} \prod_{j} \otimes \eta_{j}^{k_{j} \otimes}$,

where $\left\{\eta_{j}\right\}$ is an orthonormal system in $L^{2}(D \times T)$.

2. Multiple Wiener integrals and iterated stochastic integrals $I$.

We are going to define multiple Wiener integrals $I(F), F \in L^{2}\left((D \times T)^{n}\right)$. Let $\left\{\xi_{\alpha}\right\}$ be an orthonormal base in $H$ and let $\left\{\zeta_{\beta}\right\}$ be an orthonormal base in $L^{2}(T)$. Then $\left\{\eta_{j}\right\}$, where $\eta_{j}$ is of the form $\xi_{\alpha} \otimes \zeta_{\beta}$, is an orthonormal base in $\mathscr{H}=L^{2}(D \times T)$, and $\left\{\eta_{j_{1} \ldots j_{n}}=\eta_{j_{1}} \otimes \cdots \otimes \eta_{j_{n}}\right\}$ is an orthonormal base in $L^{2}\left((D \times T)^{n}\right)$. Therefore, if we define the multiple Wiener integrals for $\eta_{j_{1} \ldots j_{n}}$, we can extend this definition to all functions of $L^{2}\left((D \times T)^{n}\right)$. Let $B_{t}$ be the cylindrical Brownian motion on $H=L^{2}(D)$ introduced in $\S 1$. Put $F\left(x_{1}, \cdots, x_{n}\right)=\eta_{j_{1}} \otimes \cdots \otimes \eta_{j_{n}}\left(x_{1}, \cdots, x_{n}\right)$, then the multiple Wiener integral $I(F)$ of $F$ is given by the next formula

$$
I(F)=I_{n}(F)=\int \cdots \int \zeta_{\beta_{1}}\left(u_{1}\right) \cdots \zeta_{\beta_{n}}\left(u_{n}\right) d B_{u_{1}}\left(\xi_{\alpha_{1}}\right) \cdots d B_{u_{n}}\left(\xi_{\alpha_{n}}\right),
$$

where $B_{u_{k}}\left(\xi_{\alpha_{k}}\right), k=1,2, \cdots, n$ are respectively 1-dimensional Brownian motions, so the right hand side of the formula is well-defined as the usual (finite dimensional) multiple Wiener integral of degree $n$. By simple calculation we know that $I(F)$ is of the form

$$
I(F)=2^{-n / 2} \prod_{j} H_{k_{j}}\left(\left\langle\eta_{j}, \omega\right\rangle / \sqrt{2}\right), \quad \Sigma k_{j}=n,
$$

where $k_{j}$ is the multiplicity of $\eta_{j}$ in $\eta_{j_{1} \cdots j_{n}}$.

Definition 4.2. For $F, F \in L^{2}\left((D \times T)^{n}\right), I(F)$ is called the multiple Wiener integral of $F$. The set of all $I(F), F \in L^{2}\left((D \times T)^{n}\right)$, is called the space of multiple Wiener integrals of degree $n$.

From the definition of $I(F)$, we can easily prove that

$$
I(F)=I(\tilde{F}) .
$$


TheOREM 4.1. It holds that

$$
\left\{I(F): F \in L^{2}\left((D \times T)^{n}\right)\right\}=\left\{I(\hat{F}): \hat{F} \in \hat{L}^{2}\left((D \times T)^{n}\right)\right\}=\mathscr{H}_{n} .
$$

And the mapping $\left.I\right|_{\hat{L}^{2}\left((D \times T)^{n}\right)}$, the restriction of $I$, is the inverse of $\tau$.

Proof. From the formulae

$$
I\left(\eta_{j_{1} \cdots j_{n}}\right)=2^{-n / 2} \prod_{j} H_{k_{j}}\left(\left\langle\eta_{j}, \omega\right\rangle / \sqrt{2}\right), \quad \Sigma k_{j}=n
$$

and

$$
\tau\left(\prod_{j} H_{k_{j}}\left(\left\langle\eta_{j}, \omega\right\rangle / \sqrt{2}\right)\right)=\text { symmetrization of } \prod \otimes \eta_{j}^{k_{j} \otimes},
$$

it follows that $\phi=I(\tau \phi)$ for $\phi=\prod H_{k_{j}}\left(\left\langle\eta_{j}, \omega\right\rangle / \sqrt{2}\right.$ ) (note (4.4)). This equality holds true for all $\phi \in \mathscr{H}_{n}$ by the linearity of $\tau$ and $I$. (Q.E.D.)

We have obtained the following diagram:

$$
\tau: \phi \longrightarrow \tau \phi \in \hat{L}^{2}\left((D \times T)^{n}\right), \quad \phi \in \mathscr{H}_{n}, \quad \text { bijection, }
$$

$$
\begin{aligned}
& I: F \longrightarrow I(F) \in \mathscr{H}_{n}, \quad F \in L^{2}\left((D \times T)^{n}\right), \\
& \phi=I(\tau \phi), \\
& \|\phi\|_{\left(L^{2}\right) D}=(n !)^{1 / 2}\|\tau \phi\|_{L^{2}((D \times T) n)} .
\end{aligned}
$$

We will now give the definition of iterated stochastic integrals. Let $F\left(x_{1}, \cdots, x_{n}\right)$ be an element of $L^{2}\left((D \times T)^{n}\right)$. At first we assume that $F$ is a simple function. Then, for fixed $\left(x_{2}, \cdots, x_{n}\right)$ and $t_{1} F()$ is identified with an element of $L^{2}(D)=H$. So we can consider the stochastic integral (defined in $\S 1$ )

$$
\int_{-\infty}^{t_{2}}\left\langle F\left(x_{1}, x_{2}, \cdots, x_{n}\right), d B_{t_{1}}\right\rangle=\int_{-\infty}^{t_{2}}\left\langle F, d B_{t_{1}}\left(x_{1}\right)\right\rangle=\hat{I}_{1}(F)\left(x_{1}, \cdots, x_{n}\right)
$$

for all $\left(x_{2}, \cdots, x_{n}\right)$, and we have

$$
\int_{\sigma^{*}}\left|\hat{I}_{1}(F)\left(x_{2}, \cdots, x_{n}\right)\right|^{2} d \mu(\omega)=\int_{-\infty}^{t_{2}}\|F\|_{H}^{2} d t_{1} \leqq\|F\|_{L^{2}(D \times T)}^{2}
$$

For fixed $\left(x_{3}, \cdots, x_{n}\right), \hat{I}_{1}(F)$ is a function of $x_{2}$ and $\omega$, and it is $\mathscr{B}_{t_{2}}$-adapted. Considering these facts, we know that the following formula holds

$$
\begin{aligned}
& \left\|\hat{I}_{1}(F)\left(\cdot, x_{3}, \cdots, x_{n}\right)\right\|_{L^{2}\left(T \times \delta^{*} \rightarrow H\right)}^{2} \\
& \quad=\int_{D \times T}\left\|\hat{I}_{1}(F)\right\|_{L^{2}\left(\delta^{*}\right)}^{2} d x_{2} \leqq \int_{D \times T}\|F\|_{L^{2}(D \times T)}^{2} d x_{2}<\infty,
\end{aligned}
$$

for all $\left(x_{3}, \cdots, x_{n}\right)$. Therefore the stochastic integral 


$$
\hat{I}_{2}(F)\left(x_{3}, \cdots, x_{n}\right)=\int_{-\infty}^{t_{s}}\left\langle\hat{I}_{2}(F), d B_{t_{2}}\right\rangle=\int_{-\infty}^{t_{s}}\left\langle\hat{I}_{2}(F), d B_{t_{2}}\left(\boldsymbol{x}_{2}\right)\right\rangle
$$

is well-defined. Thus we are given the iterated stochastic integrals $\hat{I}_{3}(F)$, $\hat{I}_{4}(F), \cdots, \hat{I}_{n}(F)=\hat{I}(F)$, and we can easily verify the following inequality

$$
\left\|\hat{I}_{k}(F)\left(x_{k+1}, \cdots, x_{n} ; \omega\right)\right\|_{L^{2}\left((D \times T)^{\left.n-k \times e^{*}\right)}\right.}^{2} \leqq\|F\|_{L^{2}((D \times T) n)}^{2}, \quad k=1,2, \cdots, n,
$$

where we have introduced the notation $\hat{I}$ in order to discriminate the iterated stochastic integral from the multiple Wiener integral $I(F)$.

The set $\left\{F: F \in L^{2}\left((D \times T)^{n}\right)\right.$ and $F$ is a linear combination of simple functions $\}$ is dense in $L^{2}\left((D \times T)^{n}\right)$. From this and (4.9), the mapping $\hat{I}_{k}$ can be extended to be a bounded linear mapping from $L^{2}\left((D \times T)^{n}\right)$ to $L^{2}\left((D \times T)^{n-k} \times \mathscr{E}^{*}\right)$. Therefore the iterated stochastic integral $\hat{I}(F)=\hat{I}_{n}(F)$ has been defined for all $F \in L^{2}\left((D \times T)^{n}\right)$.

The following notations will be used to denote $\hat{I}_{k}(F)$ :

$$
\begin{aligned}
\hat{I}_{k}(F) & =\int^{t_{k+1}} \int^{t_{k}} \cdots \int^{t_{2}}\left\langle F, d B_{t_{1}} \cdots d B_{t_{k}}\right\rangle \\
& =\int^{t_{k+1}}\left\langle\int^{t_{k}}\left\langle\cdots \int^{t_{2}}\left\langle F, d B_{t_{1}}\right\rangle, d B_{t_{2}}\right\rangle, \cdots, d B_{t_{k}}\right\rangle \\
& =\int^{t_{k+1}}\left\langle\int^{t_{k}}\left\langle\cdots \int^{t_{2}}\left\langle F, d B_{t_{1}}\left(\boldsymbol{x}_{1}\right)\right\rangle, \cdots, d B_{t_{k}}\left(\boldsymbol{x}_{k}\right)\right\rangle\right\rangle .
\end{aligned}
$$

THEOREM 4.2. If $F \in \hat{L}^{2}\left((D \times T)^{n}\right)$, then the next formula holds

$$
I(F)=n ! \hat{I}(F) .
$$

Proof. Let $F=\eta_{i_{1}} \otimes \cdots \otimes \eta_{j_{n}}, \eta_{j_{k}}=\xi_{j_{k}} \otimes \zeta_{j_{k}}$, and put

$$
\left.\tilde{F}=\frac{1}{n !} \sum_{\pi} F^{\pi} \quad \text { (symmetrization of } F\right),
$$

where $F^{\pi}\left(x_{1}, \cdots, x_{n}\right)=F\left(x_{\pi(1)}, \cdots, x_{\pi(n)}\right)$. From the definition of $I(F)$, we obtain

$$
\begin{aligned}
I(\tilde{F}) & =I(F) \\
& =\int \cdots \int \zeta_{j_{1}}\left(u_{1}\right) \cdots \zeta_{j_{n}}\left(u_{n}\right) d B_{u_{1}}\left(\xi_{j_{1}}\right) \cdots d B_{u_{n}}\left(\xi_{j_{n}}\right) \\
& =\sum_{\pi} \int_{A_{\pi}} \cdots \int \zeta_{i_{1}}\left(u_{1}\right) \cdots \zeta_{j_{n}}\left(u_{n}\right) d B_{u_{1}}\left(\xi_{j_{1}}\right) \cdots d B_{u_{n}}^{r}\left(\xi_{j_{n}}\right) \\
\Delta \pi & =\left\{\left(u_{1}, \cdots, u_{n}\right) ; u_{\pi(1)}<u_{\pi(2)}<\cdots<u_{\pi(n)}\right\} \\
& =\sum_{\pi} \int_{u_{1}<\cdots<u_{n}} \cdots \int \zeta_{j_{\pi(1)}}\left(u_{1}\right) \cdots \zeta_{j_{\pi(n)}}\left(u_{n}\right) d B_{u_{1}}\left(\xi_{j_{\pi(1)}}\right) \cdots d B_{u_{n}}\left(\xi_{j_{\pi: n}}\right)
\end{aligned}
$$




$$
=\sum_{\pi} \hat{I}\left(F^{\pi^{-1}}\right)=\hat{I}\left(\sum_{\pi} F^{\pi^{-1}}\right)=\hat{I}(n ! \tilde{F})=n ! \hat{I}(\tilde{F}) .
$$

Since $\left\{\tilde{F} ; F\right.$ is in the form of $\left.\eta_{j_{1}} \otimes \cdots \otimes \eta_{j_{n}}\right\}$ is dense in $\hat{L}^{2}\left((D \times T)^{n}\right)$, the theorem has been proved.

CoROLlaRy 4.1. $\phi \in \mathscr{H}_{n}$ has the following representation

$$
\begin{aligned}
\phi & =I(\tau \phi)=n ! \hat{\Lambda}(\tau \phi) \\
& =n ! \int_{u_{1}<\cdots<u_{n}} \cdots \int\left\langle\tau \phi\left(x_{1}, \cdots, x_{n}\right), d B_{u_{1}} \cdots d B_{u_{n}}\right\rangle .
\end{aligned}
$$

Remark 4.1. The equality (4.12) explains the reason why we call $\tau \phi$ the kernel of the integral representation of $\phi$.

3. Hilbert space valued functionals of white noise.

Let $K$ be a real separable Hilbert space and put

$$
L^{2}\left(\mathscr{E}^{*} \longrightarrow K\right)=\left\{\Phi ; \mathscr{E}^{*} \longrightarrow K, \int_{\mathscr{E}^{*}}\|\Phi(\omega)\|_{K}^{2} d \mu(\omega)<\infty\right\} .
$$

First we shall consider the Wiener's direct sum decomposition of $L^{2}\left(\mathscr{E}^{*} \rightarrow\right.$ $K)$. Let $\psi$ be an element of $K$. Then $(\phi, \psi)_{K}$ is an element of $\left(L^{2}\right)_{D}$, so we can apply the mapping $\mathscr{T}$ (which is already defined by $(4.1)$ ) to $(\phi, \psi)_{K}$ :

$$
(\mathscr{T} \Phi)(\psi, \eta)=\left(\mathscr{T}(\Phi, \psi)_{K}(\eta)=\int_{\delta^{*}} e^{i\langle\eta, \omega\rangle}(\Phi, \psi)_{K} d \mu(\omega)\right.
$$

By (4.4), $(\Phi, \psi)_{K}$ has the integral kernel $\tau(\Phi, \psi)_{K}$ which belongs to $\hat{L}^{2}\left((D \times T)^{n}\right)$ and satisfies

$$
\left\|(\Phi, \psi)_{K}\right\|_{\left(L^{2}\right)_{D}}=(n !)^{1 / 2}\left\|\tau(\Phi, \psi)_{K}\right\|_{L^{2}\left((D \times T)^{n}\right)}
$$

Put

$$
\mathscr{H}_{n}(K)=\left\{\Phi \in L^{2}\left(\mathscr{E}^{*} \rightarrow K\right) ;(\Phi, \psi)_{K} \in \mathscr{H}_{n} \quad \text { for any } \psi \in K\right\}
$$

Then the mapping $(\Phi, \psi) \rightarrow \tau(\Phi, \psi)_{K}$, where $\Phi \in \mathscr{H}_{n}(K)$ and $\psi \in K$, gives a bounded bilinear operator from $\mathscr{H}_{n}(K) \times K$ to $\hat{L}^{2}\left((D \times T)^{n}\right)$. The mapping $\tau$ can be regarded as an operator from $\mathscr{H}_{n}(K)$ to $\mathscr{L}\left(K \rightarrow \hat{L}^{2}\left((D \times T)^{n}\right)\right)$ such that

$$
\tau \Phi: \psi \longrightarrow(\tau \Phi)(\psi)=\tau(\Phi, \psi)_{K} \in \hat{L}^{2}\left((D \times T)^{n}\right), \quad \psi \in K
$$

where $\mathscr{L}\left(K_{1} \rightarrow K_{2}\right)$ is the linear space of all bounded linear operators from $K_{1}$ to $K_{2}$. 
Theorem 4.3. For $\Phi \in \mathscr{H}_{n}(K)$ the operator $\tau \Phi: K \rightarrow \hat{L}^{2}\left((D \times T)^{n}\right)$, is of Hilbert-Schmidt type and satisfies

$$
\|\Phi\|_{L^{2}\left(\delta^{*} \rightarrow K\right)}=(n !)^{1 / 2}\|\tau \Phi\|_{H-S},
$$

where \|\|$_{H-S}$ stands for the Hilbert-Schmidt norm.

Proof. It is easy to see that $\tau \Phi$ is a bounded linear operator. The Hilbert-Schmidt norm of $\tau \Phi$ is calculated as follows. Let $\left\{e_{j}\right\}$ be an orthonormal base in $K$. Then it holds that

$$
\|\tau \Phi\|_{H-S}^{2}=\sum_{j}\left\|\tau\left(\Phi, e_{j}\right)_{K}\right\|_{L^{2}((D \times T) n)}^{2}=\sum_{j} \frac{1}{n !}\left\|\left(\Phi, e_{j}\right)_{K}\right\|_{\left(L^{2}\right)_{D}}^{2}=\frac{1}{n !}\|\Phi\|_{L^{2}\left(\sigma^{*} \rightarrow K\right)}^{2}
$$

We denote by $\sigma_{2}\left(H_{1}, H_{2}\right)$ the Hilbert space consisting of all HilbertSchmidt operators from $H_{1}$ to $H_{2}$.

CoRollary 4.2. The mapping $\tau$ can be regarded as a linear mapping from $\mathscr{H}_{n}(K)$ to $\sigma_{2}\left(K, \hat{L}^{2}\left((D \times T)^{n}\right)\right)$ and then $\tau$ is bijective. The operator norm $\|\tau\|$ of $\tau$ equals $(n !)^{-1 / 2}$.

Definition 4.3. The mapping $\tau: \mathscr{H}_{n}(K) \rightarrow \sigma_{2}\left(K, \hat{L}^{2}\left((D \times T)^{n}\right)\right)$ is called the first representation of $\mathscr{H}_{n}(K)$ and $\tau \Phi$ is called the first representation of $\Phi$.

Since $\tau \Phi$ is a Hilbert-Schmidt operator from $K$ to $\hat{L}^{2}\left((D \times T)^{n}\right),(\tau \Phi)^{*}$, the adjoint operator of $\tau \Phi$, is a Hilbert-Schmidt operator from $\hat{L}^{2}\left((D \times T)^{n}\right)$ to $K$ and $\left\|(\tau \Phi)^{*}\right\|_{H-S}=\|\tau \Phi\|_{H-S}$. We denote the isomorphism form $\mathscr{H}_{n}(K)$ to $\sigma_{2}\left(\hat{L}^{2}\left((D \times T)^{n}\right), K\right)$ by $\tau^{*}$ (that is $\left.\tau^{*} \Phi=(\tau \Phi)^{*}\right)$.

DEFINITION 4.4. The operator $\tau^{*}: \mathscr{H}_{n}(K) \rightarrow \sigma_{2}\left(\hat{L}^{2}\left((D \times T)^{n}\right), K\right)$ is called the second representation of $\mathscr{H}_{n}(K)$ and $\tau^{*} \Phi$ is called the second representation of $\Phi$.

Corollary 4.3. The mapping $\tau^{*}$ is bijective, and satisfies

$$
\|\Phi\|_{L^{2}\left(\delta^{*} \rightarrow K\right)}=\sqrt{n !}\left\|\tau^{*} \Phi\right\|_{H-S}
$$

THEOREM 4.4. It holds that

$$
L^{2}\left(\mathscr{E}^{*} \longrightarrow K\right)=\sum_{n=0}^{\infty} \oplus \mathscr{H}_{n}(K) . \quad(\text { direct sum })
$$

Proof. Let $\left\{e_{j}\right\}$ be an orthonormal base in $K$. Then any element $\Phi$ of $L^{2}\left(\mathscr{E}^{*} \rightarrow K\right)$ is expanded as $\Phi(\omega)=\sum_{j=1}^{\infty} \phi_{j}(\omega) e_{j}$, where $\phi_{j}(\omega) \in\left(L^{2}\right)_{D}$. By 
(4.4), $\phi_{j}(\omega)$ is decomposed as $\phi_{j}(\omega)=\sum \phi_{n, j}(\omega), \phi_{n, j}(\omega) \in \mathscr{H}_{n}$, to have $\Phi(\omega)$ $=\sum_{j=1}^{\infty} \sum_{n=0}^{\infty} \phi_{n, j}(\omega) e_{j}$. Put $K_{n, j}=\left\{\phi(\omega) e_{j}: \phi(\omega) \in \mathscr{H}_{n}\right\}$, then $L^{2}\left(\mathscr{E}^{*} \rightarrow K\right)=$ $\sum_{n, j} \oplus K_{n, j}$. Putting $\tilde{K}_{n}=\sum_{j} \oplus K_{n, j}$, we obtain $L^{2}\left(\mathscr{E}^{*} \rightarrow K\right)=\sum \oplus \tilde{K}_{n}$. Since the equality $\tilde{K}_{n}=\mathscr{H}_{n}(K)$ is obvious, the proof of the theorem has been complete.

(Q.E.D.)

Definition 4.5. The decomposition $L^{2}\left(\mathscr{E}^{*} \rightarrow K\right)=\sum \oplus \mathscr{H}_{n}(K)$ is called the Wiener's direct sum decomposition of $L^{2}(\mathscr{E} * \rightarrow K)$, and the second representation $\tau^{*} \Phi, \Phi \in \mathscr{H}_{n}(K)$, is called the kernel of $\Phi$.

4. Multiple Wiener integrals and iterated stochastic integrals II.

We will now define the multiple Wiener integral of $S, S \in \sigma_{2}\left(L^{2}\left((D \times T)^{n}\right)\right.$, $K)$.

Definition 4.6. For an element $S$ in $\sigma_{2}\left(L^{2}\left((D \times T)^{n}\right)\right.$, $\left.K\right)$, the multiple Wiener integral of $S$, call it $I(S)=\int \cdots \int S d B_{t_{1}} \cdots d B_{t_{n}}$, is an element of $L^{2}\left(\mathscr{E}^{*} \rightarrow K\right)$ determined by the formula

$$
(I(S), \psi)_{K}=I\left(S^{*} \psi\right) \quad \text { for any } \psi \in K,
$$

where $I\left(S^{*} \psi\right)$ is the multiple Wiener integral of $S^{*} \psi$ in the sense of Definition 4.2. The set of all $I(S), S \in \sigma_{2}\left(L^{2}\left((D \times T)^{n}\right), K\right)$, is called the space of multiple Wiener integrals of degree $n$.

Remark 4.2. If $\hat{S} \in \sigma_{2}\left(\hat{L}^{2}\left((D \times T)^{n}\right), K\right)$, then $\hat{S}^{*} \psi \in \hat{L}^{2}\left((D \times T)^{n}\right)$ for $\psi$ $\in K$. Therefore an element in $L^{2}\left(\mathscr{E}^{*} \rightarrow K\right)$ is uniquely determined by

$$
(I(\hat{S}), \psi)_{K}=I\left(\hat{S}^{*} \psi\right) \quad \text { for any } \psi \in K .
$$

$I(\hat{S})=\int \cdots \int \hat{S} d B_{t_{1}} \cdots d B_{t_{n}}$ is called the multiple Wiener integral of $\hat{S}$.

TheOREM 4.5. (i) For $S \in \sigma_{2}\left(L^{2}\left((D \times T)^{n}\right), K\right)$ put $\hat{S}=\left.S\right|_{\hat{L}^{2}\left((D \times T)^{n}\right)}$ (the restriction of $S$ to $\left.\hat{L}^{2}\left((D \times T)^{n}\right)\right)$. Then it holds that $I(S)=I(\hat{S})$.

(ii) The operator $I: \sigma_{2}\left(L^{2}\left((D \times T)^{n}\right), K\right) \rightarrow L^{2}\left(\mathscr{E}^{*} \rightarrow K\right)$ is linear and bounded. In addition, for $S \in \sigma_{2}\left(\hat{L}^{2}\left((D \times T)^{n}\right), K\right)$, we have

$$
\|I(S)\|_{L^{2}\left(\mathscr{G}^{*} \rightarrow K\right)}=(n !)^{1 / 2}\|S\|_{H-S} .
$$

Proof. (i) We will first prove that

$$
\hat{S}^{*} \psi=\widetilde{S^{*} \psi} \quad \text { for any } \psi \in K,
$$

where $\sim$ stands for the symmetrization. This equality comes from the next 
formulae, for $f \in \hat{L}^{2}\left((D \times T)^{n}\right)$

$$
\begin{aligned}
\left(\widetilde{S^{*} \psi}, f\right)_{L^{2}} & =\frac{1}{\mathrm{n} !} \sum_{\pi}\left(\left(S^{*} \psi\right)^{\pi}, f\right)_{L^{2}}=\frac{1}{n !} \sum_{\pi}\left(S^{*} \psi, f\right)_{L^{2}} \\
& =\left(S^{*} \psi, f\right)_{L^{2}}=(\psi, S f)_{K}
\end{aligned}
$$

and

$$
\left(\hat{S}^{*} \psi, f\right)_{L^{2}}=(\psi, \hat{S} f)_{K}=(\psi, S f)_{K}
$$

From (4.5) and (4.22) we obtain

$$
(I(S), \psi)_{K}=I\left(S^{*} \psi\right)=I\left(\overparen{S^{*} \psi}\right)=I\left(\hat{S}^{*} \psi\right)=(I(\hat{S}), \psi)_{K},
$$

which is to be proved.

(ii) Let $\left\{e_{j}\right\}$ be an orthonormal base in $K$. Then, from (4.6) we have

$$
\begin{aligned}
\int_{\delta^{*}}\|I(S)\|^{2} d \mu(\omega) & =\int_{\delta *} \sum_{j}\left|\left(I(S), e_{j}\right)\right|^{2} d \mu(\omega) \\
& =\sum_{j} \int_{\delta^{*}}\left|I\left(S^{*} e_{j}\right)\right|^{2} d \mu(\omega)=\sum_{j} n !\left\|S^{*} e_{j}\right\|_{L^{2}}^{2} \\
& =n !\left\|S^{*}\right\|_{H-S}^{2}=n !\|S\|_{H-S}^{2} .
\end{aligned}
$$

The rest of Theorem is trivial.

THEOREM 4.6. Let $\Phi(\omega)$ be an element of $\mathscr{H}_{n}(K)$. Then $\Phi$ is represented as the multiple Wiener integral of $\tau^{*} \Phi$, that is

$$
\Phi(\omega)=I\left(\tau^{*} \Phi\right)=\int \cdots \int \tau^{*} \Phi d B_{t_{1}} \cdots d B_{t n} .
$$

Proof. Since the inner product $(\Phi, \psi), \psi \in K$, belongs to $\mathscr{H}_{n}$, we obtain

$$
(\Phi, \psi)_{K}=I\left(\tau(\Phi, \psi)_{K}\right)=I((\tau \Phi) \psi)
$$

On the other hand it holds that

$$
\left(I\left(\tau^{*} \Phi\right), \psi\right)_{K}=I\left(\left(\tau^{*} \Phi\right)^{*} \psi\right)=I\left(\left((\tau \Phi)^{*}\right)^{*} \psi\right)=I((\tau \Phi) \psi)
$$

Therefore we have proved the Theorem.

Let $B_{t}$ be the cylindrical Brownian motion on $H=L^{2}(D)$ introduced in $\S 1$. Then the stochastic integral $\int_{T} S(t) d B_{t}$ is defined, where $S$ is $\mathscr{B}_{t^{-}}$ adapted and $S \in L^{2}\left(T \times \mathscr{E}^{*} \rightarrow \sigma_{2}(H, K)\right)$. The integral $\int_{T} S(t) d B_{t}$ is an ele- 
ment of $L^{2}\left(\mathscr{E}^{*} \rightarrow K\right)$. We will treat the iteration of stochastic integral of this type.

Let $S \in \sigma_{2}(H, K)$, then $S$ is represented as

$$
S \xi=\sum_{j} \lambda_{j}\left(\xi, \xi_{j}\right) e_{j}=\int_{D} F_{S}(\boldsymbol{x}) \xi(\boldsymbol{x}) d \boldsymbol{x}
$$

where $\left\{\xi_{j}\right\}$ is an orthonormal base in $H,\left\{e_{j}\right\}$ is an orthonormal system of $K, \sum \lambda_{j}^{2}=\|S\|_{H-S}^{2}<\infty$, and $F_{S}(\boldsymbol{x})=\sum_{j} \lambda_{j} \xi_{j}(\boldsymbol{x}) e_{j}\left(F_{S}: D \rightarrow K\right)$. Since $F_{S}$ satisfies

$$
\int_{D}\left\|F_{S}\right\|_{K}^{2} d x=\sum_{j} \int \lambda_{j}^{2} \xi_{j}^{2}(x) d x=\sum_{j} \lambda_{j}^{2}=\|S\|_{H-S}^{2}<\infty,
$$

$F_{S}$ belongs to $L^{2}(D \rightarrow K)$.

Proposition 4.1. The above correspondence of $S$ and $F_{S}$ determines an isomorphism between $\sigma_{2}(H, K)$ and $L^{2}(D \rightarrow K)$.

Proof. It is already proved that $F_{S} \in L^{2}(D \rightarrow K)$ and $\left\|F_{S}\right\|=\|S\|_{H-S}$ for any $S \in \sigma_{2}(H, K)$. Let $F \in L^{2}(D \rightarrow K)$ and put $S_{F}$ the operator defined by

$$
S_{F} \xi=\int_{D} F(\boldsymbol{x}) \xi(\boldsymbol{x}) d \boldsymbol{x}, \quad \xi \in H .
$$

Then $S_{F}: H \rightarrow K$ is well-defined and is a bounded linear operator. The Hilbert-Schmidt norm of $S_{F}$ is equal to

$$
\begin{aligned}
\sum_{j}\left\|S_{F} \xi_{j}\right\|_{K}^{2} & =\sum_{j} \sum_{i}\left|\left(S_{F} \xi_{j}, e_{i}\right)_{K}\right|^{2} \\
& =\sum_{i, j}\left|\int_{D}\left(F(\boldsymbol{x}), e_{i}\right)_{K} \cdot \xi_{j}(\boldsymbol{x}) d \boldsymbol{x}\right|^{2} \\
& =\sum_{i} \int_{D}\left|\left(F(\boldsymbol{x}), e_{i}\right)_{K}\right|^{2} d \boldsymbol{x} \\
& =\|F\|_{L^{2}(D \rightarrow K)}^{2} .
\end{aligned}
$$

Thus the proof is completed.

In the same manner we can prove the next proposition.

Proposition 4.2. The following diagrams are true.

$$
\begin{aligned}
& \sigma_{2}\left(L^{2}\left(D^{n}\right), K\right) \cong L^{2}\left(D^{n} \rightarrow K\right) \\
& \sigma_{2}\left(\hat{L}^{2}\left(D^{n}\right), K\right) \cong \hat{L}^{2}\left(D^{n} \rightarrow K\right) \\
& \sigma_{2}\left(L^{2}\left((D \times T)^{n}\right), K\right) \cong L^{2}\left((D \times T)^{n} \rightarrow K\right)
\end{aligned}
$$




$$
\sigma_{2}\left(\hat{L}^{2}\left((D \times T)^{n}\right), K\right) \cong \hat{L}^{2}\left((D \times T)^{n} \rightarrow K\right)
$$

where $\cong$ denotes isomorphism.

Proposition 4.3. (i) We have the following diagram

$$
\begin{aligned}
L^{2}\left(\mathscr{E}^{*} \longrightarrow \sigma_{2}\left(L^{2}(D \times T), K\right)\right) & \cong L^{2}\left(\mathscr{E}^{*} \longrightarrow L^{2}((D \times T) \longrightarrow K)\right) \\
L^{2}\left(T \times \mathscr{E}^{*} \longrightarrow \sigma^{2}\left(L^{2}(D), K\right)\right. & \cong L^{2}\left(T \times \mathscr{E}^{*} \longrightarrow L^{2}(D \longrightarrow K)\right) \\
& \cong L^{2}\left(\mathscr{E}^{*} \times D \times T \longrightarrow K\right)
\end{aligned}
$$

(ii) If $S \in L^{2}\left(T \times \mathscr{E}^{*} \rightarrow \sigma_{2}\left(L^{2}(D), K\right)\right)$ and $S$ is $\mathscr{B}_{t}$-adapted, then the stochastic integral $\int_{T} S(t, \omega) d B_{t}$ is well-defined. Put $\hat{I}(S)=\int_{T} S(t) d B_{t}$, then

$$
E\left[\|\hat{I}(S)\|_{K}^{2}\right]=\int E\left[\|S\|_{H-S}^{2}\right] d t=\int_{D \times T} E\left[\left\|F_{S}\right\|_{K}^{2}\right] d x
$$

where $F_{S}$ is the element of $L_{2}\left(\mathscr{E}^{*} \times D \times T \rightarrow K\right)$ corresponding to $S$ by (4.24).

Proof. (i) is easily proved by the use of Proposition 4.2. (ii) is obvious from the definition of the stochastic integral and Proposition 4.1.

(Q.E.D.)

Remark 4.3. We identify the elements of $L^{2}\left(T \times \mathscr{E}^{*} \rightarrow \sigma_{2}\left(L^{2}(D), K\right)\right)$ with those of $L^{2}\left(\mathscr{E}^{*} \times D \times T \rightarrow K\right)$ and we use the notation $\int_{T} S(t, \omega) d B_{t}$ or $\int_{T} S(t, \omega ; \boldsymbol{x}) d B_{t}(\boldsymbol{x})$ to indicate the stochastic integral $\hat{I}(S)$. We also use the notation $S\left(x_{1}, \cdots, x_{n}\right)$ instead of $F_{S}\left(x_{1}, \cdots, x_{n}\right)$.

We are now ready to define the iterated stochastic integrals. Let $S \in$ $\sigma_{2}\left(L^{2}\left((D \times T)^{n}\right), K\right)$, then $S$ is considered as an element of $L^{2}\left((D \times T)^{n} \rightarrow K\right)$ by Proposition 4.2. We assume here that $S$ is a simple functional. Once $\left(x_{2}, \cdots, x_{n}\right)$ is fixed, we can regard $S$ as an element of $L^{2}(D \times T \rightarrow K)$. While $S$ is also regarded as an element of $\sigma_{2}\left(L^{2}(D \times T), K\right)$ or $L^{2}(T \rightarrow$ $\left.\sigma_{2}\left(L^{2}(D), K\right)\right)$ by Proposition $4.3(\mathrm{i})$, and this function is denoted by $S^{x_{2} \cdots r n}\left(t_{1}\right)$. Thus we know that the stochastic integral

$$
\hat{I}_{1}(S)\left(x_{2}, \cdots, x_{n}\right)=\int_{-\infty}^{t_{2}} S^{x_{2} \cdots x_{n}}\left(t_{1}\right) d B_{t_{1}}
$$

is well-defined in the sense of Definition 1.4 and we have

$$
E\left\|\hat{I}_{1}(S)\left(x_{2}, \cdots, x_{n}\right)\right\|_{K}^{2}=\int^{t_{2}}\left\{\int_{D}\left\|S\left(x_{1}, \cdots, x_{n}\right)\right\|_{K}^{2} d x_{1}\right\} d t_{1} \leqq \int_{D \times T}\|S\|_{K}^{2} d x_{1} .
$$

From this inequality we obtain 


$$
\begin{aligned}
& \int_{(D \times T)^{n-1}} \cdots \int E\left\|\hat{I}_{1}(S)\left(x_{2}, \cdots, x_{n}\right)\right\|_{K}^{2} d x_{2} \cdots d x_{n} \leqq \int_{(D \times T)^{n}} \int\|S\|_{K}^{2} d x_{1} \cdots d x_{n}, \\
& \hat{I}_{1}(S) \in L^{2}\left(\mathscr{E}^{*} \longrightarrow L^{2}\left((D \times T)^{n-1} \longrightarrow K\right)\right) \cong L^{2}\left(\mathscr{E}^{*} \longrightarrow \sigma_{2}\left(L^{2}\left((D \times T)^{n-1}, K\right)\right) .\right.
\end{aligned}
$$

Using Proposition 4.3, we can define the stochastic integral

$$
\hat{I}_{2}(S)\left(x_{3}, \cdots, x_{n}\right)=\int^{t_{3}} \hat{I}_{1}(S)\left(x_{2}, \cdots, x_{n}\right) d B_{t_{2}}\left(x_{2}\right)
$$

which has the same properties as $\hat{I}_{1}(S)\left(x_{2}, \cdots, x_{n}\right)$ described above. Repeating this procedure $n$ times, we finally arrive at the iterated stochastic integral

$$
\hat{I}(S)=\int\left\{\int^{t_{n}}\left\{\cdots\left\{\int^{t_{2}} S^{x_{2} \cdots r_{n}}\left(t_{1}\right) d B_{t_{1}}\right\} d B_{t_{2}} \cdots\right\} d B_{t_{n-1}}\right\} d B_{t_{n}},
$$

and $\hat{I}(S)$ satisfies

$$
\|\hat{I}(S)\|_{L^{2}\left(\sigma^{*} \rightarrow K\right)}^{2} \leqq \int_{(D \times T)^{n}} \cdots \int\|S\|_{K}^{2} d x_{1} \cdots d x_{n}=\|S\|_{H-S}^{2} \cdot
$$

Since the set $\left\{S: S \in L^{2}\left((D \times T)^{n} \rightarrow K\right), S\right.$ is a linear combination of simple functions $\}$ is dense in $L^{2}\left((D \times T)^{n} \rightarrow K\right)$, the mapping $\hat{I}: S \rightarrow \hat{I}(S)$, can be extended to be a bounded linear mapping from $L^{2}\left((D \times T)^{n} \rightarrow K\right)(\cong$ $\left.\sigma_{2}\left(L^{2}\left((D \times T)^{n}\right), K\right)\right)$ to $L^{2}\left(\mathscr{E}^{*} \rightarrow K\right)$.

Definition 4.7. The $K$-valued random variable $\hat{I}(S)$, which has just been obtained above, is called the iterated stochastic integral of $S$.

$\hat{I}(S)$ is also denoted by

$$
\int_{t_{1} \leqq \cdots \leqq t_{n}} \cdots \int S\left(x_{1}, \cdots, x_{n}\right) d B_{t_{1}}\left(\boldsymbol{x}_{1}\right) \cdots d B_{t_{n}}\left(\boldsymbol{x}_{n}\right)
$$

or simply by $\int_{t_{1} \leqq \cdots \leqq t_{n}} \cdots \int S d B_{t_{1}} \cdots d B_{t_{n}}$.

Remark 4.4. If $\hat{S} \in \sigma_{2}\left(\hat{L}^{2}\left((D \times T)^{n}\right), K\right) \cong \hat{L}^{2}\left((D \times T)^{n} \rightarrow K\right)$, then $\hat{S}$ can be regarded as an element of $L^{2}\left((D \times T)^{n} \rightarrow K\right)$. So the iterated stochastic integral $\hat{I}(\hat{S})$ is well-defined.

Proposition 4.4. Let $S \in \sigma_{2}\left(L^{2}\left((D \times T)^{n}\right), K\right)$ and put $\hat{S}=\left.S\right|_{\hat{L}^{2}\left((D \times T)^{n}\right)}$. Then $\hat{S} \in \sigma_{2}\left(\hat{L}^{2}\left((D \times T)^{n}\right), K\right)$ and $F_{\hat{S}}=\tilde{F}_{S}\left(=\right.$ the symmetrization of $\left.F_{S}\right)$.

Proof. For any $f \in \hat{L}^{2}\left((D \times T)^{n}\right)$ it holds that

$$
S f=\int \cdots \int F_{S}\left(x_{1}, \cdots, x_{n}\right) f\left(x_{1}, \cdots, x_{n}\right) d x_{1} \cdots d x_{n}
$$




$$
\begin{aligned}
& =\int \cdots \int F_{S}^{\pi}\left(x_{1}, \cdots, x_{n}\right) f\left(x_{1}, \cdots, x_{n}\right) d x_{1} \cdots d x_{n} \\
& =\int \cdots \int \tilde{F}_{S}\left(x_{1}, \cdots, x_{n}\right) f\left(x_{1}, \cdots, x_{n}\right) d x_{1} \cdots d x_{n} .
\end{aligned}
$$

Therefore we obtain $F_{\hat{S}}=\tilde{F}_{s}$.

(Q.E.D.)

We will finally discuss the connection between $I(S)$ and $\hat{I}(S)$.

THeOREM 4.7. It holds that

$$
\left.I(S)=n ! \hat{I}(S) \quad \text { for any } S \in \sigma_{2}\left((D \times T)^{n}\right), K\right) .
$$

Proof. Let $S \in \sigma_{2}\left(L^{2}\left((D \times T)^{n}\right), K\right)$, then by Proposition 4.2 there is a function $F_{S} \in L^{2}\left((D \times T)^{n} \rightarrow K\right)$ such that

$$
S f=\int \cdots \int F_{S}\left(x_{1}, \cdots, x_{n}\right) f\left(x_{1}, \cdots, x_{n}\right) d x_{1} \cdots d x_{n}, f \in L^{2}\left((D \times T)^{n}\right) .
$$

Define the operator $S^{x_{2} \cdots x_{n}} \in \sigma_{2}\left(L^{2}(D \times T), K\right)$ by

$$
S^{x_{2} \cdots x_{n}} \eta=\int F_{S}\left(x_{1}, x_{2}, \cdots, x_{n}\right) \eta\left(x_{1}\right) d x_{1} .
$$

Then $S^{x_{2} \cdots x_{n}}$ is well-defined for almost all $\left(x_{2}, \cdots, x_{n}\right)$, and the next equality holds

$$
\begin{aligned}
\left(\left(S^{x_{2} \cdots x_{n}}\right)^{*} \psi, \eta\right)_{L^{2}(D \times T)} & =\left(\psi, S^{x_{2} \cdots x_{n}} \eta\right)_{K} \\
& =\left(\psi, \int F_{S}\left(x_{1}, x_{2}, \cdots, x_{n}\right) \eta\left(x_{1}\right) d x_{1}\right)_{K} \\
& =\int\left(\psi, F_{S}\left(x_{1}, x_{2}, \cdots, x_{n}\right)\right)_{K} \eta\left(x_{1}\right) d x_{1}
\end{aligned}
$$

for $\psi \in K$ and $\eta \in L^{2}(D \times T)$. We can regard $\left(\left(S^{x_{2} \cdots x_{n}}\right) * \psi\right)\left(x_{1}\right)$ as an element of $L^{2}\left((D \times T)^{n}\right)$, and then we have

$$
\begin{aligned}
& \left(\left(\left(S^{x_{2} \cdots x_{n}}\right)^{*} \psi\right)\left(x_{1}\right), \eta \otimes g\right)_{L^{2}((D \times T) n)} \\
& \quad=\int_{(D \times T)^{n-1}} \cdots \int\left(\left(S^{x_{2} \cdots x_{n}}\right)^{*} \psi, \eta\right)_{L^{2}(D \times T)} g\left(x_{2}, \cdots, x_{n}\right) d x_{2} \cdots d x_{n} \\
& \quad=\int_{(D \times T)^{n}} \cdots \int\left(\psi, F_{S}\right)_{K} \eta\left(x_{1}\right) g\left(x_{2}, \cdots, x_{n}\right) d x_{1} \cdots d x_{n}
\end{aligned}
$$

for $g \in L^{2}\left((D \times T)^{n-1}\right)$. On the other hand it holds that

$$
\begin{aligned}
& \left(S^{*} \psi, \eta \otimes g\right)_{L^{2}\left((D \times T)^{n)}\right.}=\left(\psi, S^{*}(\eta \otimes g)\right)_{K} \\
& \quad=\left(\psi, \int \cdots \int F_{S}\left(x_{1}, \cdots, x_{n}\right) \eta\left(x_{1}\right) g\left(x_{2}, \cdots, x_{n}\right) d x_{1} \cdots d x_{n}\right)_{K}
\end{aligned}
$$




$$
=\int \cdots \int\left(\psi, F_{S}\right) \eta\left(x_{1}\right) g\left(x_{2}, \cdots, x_{n}\right) d x_{1} \cdots d x_{n} .
$$

From (4.28) and (4.29) we obtain

$$
\left(\left(S^{x_{2} \cdots x_{n}}\right)^{*} \psi\right)\left(x_{1}\right)=\left(S^{*} \psi\right)\left(x_{1}, \cdots, x_{n}\right) \quad \text { in } L^{2}\left((D \times T)^{n}\right),
$$

and so we know that for almost all $\left(x_{2}, \cdots, x_{n}\right)$

$$
\left(S^{x_{2} \cdots x_{n}}\right)^{*} \psi=\left(S^{*} \psi\right)\left(\cdot, x_{2}, \cdots, x_{n}\right)
$$

in $L^{2}(D \times T)$ sense. From this equality it follows that

$$
\begin{aligned}
& \left(\psi, \hat{I}_{1}(S)\left(x_{2}, \cdots, x_{n}\right)\right)_{K} \\
& \quad=\int^{t_{2}}\left\langle\left(\left(S^{x_{2} \cdots x_{n}}\right)^{*} \psi\right)\left(x_{1}\right), d B_{t_{1}}\left(\boldsymbol{x}_{1}\right)\right\rangle \\
& \quad=\int^{t_{2}}\left\langle\left(S^{*} \psi\right)\left(x_{1}, \cdots, x_{n}\right), d B_{t_{1}}\left(\boldsymbol{x}_{1}\right)\right\rangle
\end{aligned}
$$

for almost all $\left(x_{2}, \cdots, x_{n}\right)$.

Now we shall prove inductively that

$$
\left(\psi, \hat{I}_{k}(S)\right)_{K}=\int^{t_{k+1}}\left\langle\int \cdots \int^{t_{2}}\left\langle S^{*} \psi, d B_{t_{1}}\right\rangle, \cdots, d B_{t_{k}}\right\rangle
$$

for almost all $\left(x_{k+1}, \cdots, x_{n}\right), k=1,2, \cdots, n$. Assume that (4.31) is proved for $k-1$. Then, by the definition of $\hat{I}_{k-1}(S)$, for any $\eta \in L^{2}(D \times T)$ we have

$$
\begin{aligned}
& \left(\hat{I}_{k-1}(S) * \psi, \eta\right)_{L^{2}(D \times T)}=\left(\psi, \hat{I}_{k-1}(S) \eta\right)_{K} \\
& \quad=\int_{D \times T}\left(\psi, \hat{I}_{k-1}(S)\left(x_{k}, \cdots, x_{n}\right)\right)_{k} \eta\left(x_{k}\right) d x_{k} \\
& \quad=\left(\left(\psi, \hat{I}_{k-1}(S)\right)_{K}, \eta\right)_{L^{2}(D \times T)} .
\end{aligned}
$$

Using the assumption of induction and (4.32), we know that

$$
\hat{I}_{k-1}(S)^{*} \psi=\int^{t_{k}}\left\langle\cdots \int^{t_{2}}\left\langle S^{*} \psi, d B_{t_{1}}\right\rangle, \cdots, d B_{t_{k-1}}\right\rangle,
$$

so we obtain

$$
\begin{aligned}
\left(\psi, \hat{I}_{k}(S)\right)_{K} & =\int^{t_{k+1}}\left\langle\hat{I}_{k-1}(S)^{*} \psi, d B_{t_{k}}\right\rangle \\
& =\int^{t_{k+1}}\left\langle\left\{\int^{t_{k}}\left\langle\cdots \int^{t_{2}}\left\langle S^{*} \psi, d B_{t_{1}}\right\rangle, \cdots, d B_{t_{k-1}}\right\rangle\right\}, d B_{t_{k}}\right\rangle,
\end{aligned}
$$

which is to be proved.

Putting $k=n$ in the formula (4.31), we obtain 


$$
(\psi, \hat{I}(S))_{K}=\hat{I}\left(S^{*} \psi\right)
$$

Let $S \in \sigma_{2}\left(\hat{L}^{2}\left((D \times T)^{n}\right), K\right)$, then $S^{*} \psi \in \hat{L}^{2}\left((D \times T)^{n}\right)$. By Theorem 4.2 we have

$$
(\psi, I(S))_{K}=I\left(S^{*} \psi\right)=n ! \hat{I}\left(S^{*} \psi\right)=n !(\psi, \hat{I}(S))_{K}
$$

for any $\psi \in K$. Thus we have proved (4.27).

Summing up what have been discussed, we have obtained the following diagram:

$$
\begin{aligned}
& L^{2}\left(\mathscr{E}^{*} \longrightarrow K\right)=\sum_{n=0}^{\infty} \oplus \mathscr{H}_{n}(K), \\
& \mathscr{H}{ }_{n}(K) \cong \sqrt{n !} \sigma_{2}\left(\hat{L}^{2}\left((D \times T)^{n}\right), K\right) \cong \sqrt{n !} \hat{L}^{2}\left((D \times T)^{n} \longrightarrow K\right), \\
& \tau^{*}: \Phi \longrightarrow \tau^{*} \Phi \in \sigma_{2}\left(\hat{L}^{2}\left((D \times T)^{n}\right), K\right) \cong \hat{L}^{2}\left((D \times T)^{n} \longrightarrow K\right), \\
& \Phi \in \mathscr{H}_{n}(K), \text { bijection }, \\
& I: F \longrightarrow I(F)=n ! \hat{I}(F)=\int_{t_{1} \leqq \cdots \leqq t_{n}} \int \begin{array}{c}
F d B_{t_{1}} \cdots d B_{t_{n}}, \\
F \in \hat{L}^{2}\left((D \times T)^{n} \longrightarrow K\right),
\end{array} \\
& I \cdot \tau^{*}=\text { identity . }
\end{aligned}
$$

Before closing this section, we mention an interesting result in connection with the stochastic differential equation (3.2) in $\S 3$. Since the unique solution $\tilde{X}_{t}$ of (3.2) is an element of $L^{2}\left(\mathscr{E}^{*} \rightarrow \tilde{H}_{-1 / 2}\right), \tilde{X}_{t}$ has a kernel of integral representation.

THEOREM 4.8. The kernel of the integral representation of $\tilde{X}_{t}$ with $\hat{X}_{0}$ $=0$ is given by

$$
\chi_{[0, t]}(u) \sum_{j=1}^{\infty} e^{-j(t-u)} \xi_{j}(\sigma) \xi_{j} .
$$

Proof. This result follows from the above discussions without any difficulties.

(Q.E.D.)

\section{§5. Stochastic differential equations with multiplicative operator}

This section is devoted to a development of our theory. Actually we shall discuss the so-called bilinear stochastic differential equation on the Hilbert space $H=L^{2}([0, \pi])$ given by

$$
d X_{t}=-\hat{\omega} X_{t} d t+X_{t} \cdot d B_{t},
$$

where $\hat{\omega}(=-\sqrt{-\Delta})$ was given in $\S 3$ and $X_{t}$. denote the multiplicative 
operator, i.e. $\left(X_{t} \cdot \xi\right)(\sigma)=X_{t}(\sigma) \xi(\sigma), \sigma \in[0, \pi]$.

Although our techniques developed in $\S 3$ are not available to the equation of this type, our results established in $\S 4$ do work in the investigation of the equation above. The reason could be seen in the discussions what follow.

Before we come to details, we have to overcome a difficulty. Namely, there is no solution to (5.1) living even in $H_{-\infty}$ formed by the Hilbert scale derived from $\hat{\omega}$. To avoid this difficulty, we are led to consider a modified equation (5.4) under the assumption (5.5) (note that if $\Gamma\left(\sigma, \sigma^{\prime}\right)=\delta\left(\sigma-\sigma^{\prime}\right)$, then (5.4) turns into (5.1)), which will be prescribed later.

We first investigate some properties of the multiplicative operator. Let $A$ be a positive self-adjoint operator on $H$ determined by

$$
\begin{aligned}
& A \xi_{0}=\xi_{0} \\
& A \xi_{j}=\hat{\omega} \xi_{j}=j \xi_{j}, \quad j=1,2, \cdots,
\end{aligned}
$$

where $\left\{\xi_{j}\right\}, j=0,1,2, \cdots$, is the orthonormal base for $H$ given in $\S 3$ (i.e. $\xi_{0}=\pi^{-1 / 2}$ and $\left.\xi_{j}=(2 / \pi)^{1 / 2} \cos j \sigma\right)$. Since $A^{-1}$ is of Hilbert-Schmidt type, a Hilbert scale $\left\{H_{\alpha}\right\},-\infty<\alpha<\infty$, is generated by $A$, where $H_{\alpha}$ is a Hilbert space with an inner product $(\xi, \eta)_{\alpha}=\left(A^{\alpha} \xi, A^{\alpha} \eta\right)_{H}$.

Put $T_{t}=e^{-t \hat{\theta}}, t \geqq 0,\left(T_{t}\right.$ is the same operator as given in $\S 3$, but the domain is $H$, not $\tilde{H}$ ). Then we have

Proposition 5.1. (i) The multiplicative operator $X \cdot X \in H$, is not bounded on $H$. If $t>0$, then the closed extension of $T_{t} X$. is a HilbertSchmidt operator on $H$.

(ii) The multiplicative operator $X \cdot X \in H$, is considered as a bounded operator from $H$ into $H_{-\alpha}, \alpha>1 / 2$. Moreover it holds that $X \cdot \in \sigma_{2}\left(H, H_{-\alpha}\right)$ and that

$$
\|X \cdot\|_{\sigma_{2}(H, H-\alpha)}^{2} \leqq \text { const. }\left(1+\sum_{k=1}^{\infty} k^{-2 \alpha}\right)\|X\|^{2} .
$$

(iii) If $X \in H_{-\alpha}$ for some $\alpha>0$ but $X \notin H$, then $T_{t} X \cdot \notin \sigma_{2}\left(H, H_{-\beta}\right)$ however large $\beta$ may be chosen.

Remark 5.1. For $X \in H T_{t} X$. is of Hilbert-Schmidt type by Proposition 5.1 (i). But the integration $\int_{0}^{t}\left\|T_{t-S} X \cdot\right\|_{\sigma_{2}(H)}^{2} d s$ does not always converge. For example, if $X \neq 0$ and $a_{n} \geqq 0$ in the expansion $X=\sum a_{n} \xi_{n}$, then $\int_{0}^{t}\left\|T_{t-s} X \cdot\right\|_{\sigma_{2}(H)}^{2} d s$ diverges. 
Proof of Proposition 5.1. (i) Take an element $X$ in $H=L^{2}([0, \pi])$ such that $X \notin L^{4}([0, \pi])$. Then $X \cdot X=X^{2} \notin H$, i.e. $X$. is not a bounded operator on $H$. Having the $X$ expressed in the form $X=\sum a_{n} \xi_{n}$, we obtain

$$
\begin{aligned}
\sum_{m=0}^{\infty}\left\|T_{t}\left(X \xi_{m}\right)\right\|^{2} & =\sum_{m} \sum_{k}\left(T_{t}\left(X \xi_{m}\right), \xi_{k}\right)^{2} \\
& =\sum_{m} \sum_{k}\left(\sum_{n} a_{n} e^{-k t}\left(\xi_{n} \xi_{m}, \xi_{k}\right)\right)^{2} \\
& \leqq 2 \pi^{-2} \sum_{k} e^{-2 k t} \sum_{m}\left(a_{m+k}^{2}+a_{|m-k|}^{2}\right) \\
& \left.\leqq 6 \pi^{-2}\|X\|^{2} \sum e^{-2 k t}<\infty \quad \text { (if } t>0\right),
\end{aligned}
$$

where we have used the following formula

$$
\sum_{n} a_{n}\left(\xi_{n} \xi_{m}, \xi_{k}\right)= \begin{cases}\frac{1}{\pi}\left(a_{m+k}+a_{|m-k|}\right), & \text { if } m, k \geqq 1, \\ \frac{1}{\sqrt{2} \pi}\left(a_{m+k}+a_{|m-k|}\right), & \text { if } m k=0, \quad m+k \geqq 1, \\ \frac{1}{\pi} a_{0}, & \text { if } m=k=0 .\end{cases}
$$

The inequality (5.2) proves that the closed extension of $T_{t} X$. is an element of $\sigma_{2}(H)$.

(ii) With the expression $X=\sum a_{n} \xi_{n}$, we obtain

$$
\begin{aligned}
\|X \cdot\|_{\sigma_{2}(H, H-\alpha)}^{2} & =\sum_{m}\left\|X \cdot \xi_{m}\right\|_{-\alpha}^{2}=\sum_{k, m}\left(X \cdot \xi_{m}, \xi_{k}\right)_{-\alpha}^{2} \\
& =\sum_{k, m}\left(\sum a_{n}\left(\xi_{n} \xi_{m}, \xi_{k}\right)_{-\alpha}\right)^{2} \\
& =\sum_{m}\left(\sum_{n} a_{n}\left(\xi_{n} \xi_{m}, \xi_{0}\right)\right)^{2}+\sum_{k \geqq 1} \sum_{m} k^{-2 \alpha}\left(\sum_{n} a_{n}\left(\xi_{n} \xi_{m}, \xi_{k}\right)\right)^{2} \\
& \leqq 4 \pi^{-2} \sum_{m} a_{m}^{2}+\pi^{-2} \sum_{k \geqq 1} k^{-2 \alpha} \sum_{m}\left(a_{m+k}+a_{|m-k|}\right)^{2} \\
& \leqq 6 \pi^{-2}\|X\|^{2}\left(1+\sum_{k \geqq 1} k^{-2 \alpha}\right) .
\end{aligned}
$$

(iii) Suppose that $\sum a_{n}^{2}=\infty, \sum n^{-2 \alpha} a_{n}^{2}<\infty$ and that $a_{n} \geqq 0$ in the expansion $X=\sum a_{n} \xi_{n}$. If $T_{t} X$. were well-defined and $T_{t} X \cdot \in \sigma_{2}\left(H, H_{-\beta}\right)$, then the following formula should hold

$$
\begin{aligned}
\left\|T_{t} X \cdot\right\|_{\sigma_{2}(H, H-\beta)}^{2} & =\sum_{m}\left\|T_{t} X \xi_{m}\right\|_{-\beta}^{2} \\
& =\sum_{m}\left\{\left(T_{t} X \xi_{m}, \xi_{0}\right)^{2}+\sum_{k \geqq 1}\left(T_{t} X \xi_{m}, k^{\beta} \xi_{k}\right)_{-\beta}^{2}\right\} \\
& =\sum_{m}\left\{\left(\sum_{n} a_{n}\left(\xi_{n} \xi_{m}, \xi_{0}\right)\right)^{2}+\sum_{k \geqq 1} k^{-2 \beta} e^{-2 k t}\left(\sum_{n} a_{n}\left(\xi_{r} \xi_{m}, \xi_{0}\right)\right)^{2}\right\}
\end{aligned}
$$




$$
\begin{aligned}
& \geqq\left(1 / 4 \pi^{2}\right)\left\{\sum_{m} a_{m}^{2}+\sum_{k \geqq 1}\left(k^{-2 \beta} e^{-2 k t} \sum_{m}\left(a_{m+k}^{2}+a_{|m-k|}^{2}\right)\right)\right\} \\
& \geqq\left(1 / 4 \pi^{2}\right)\left(1+\sum_{k \geq 1} k^{-2 \beta} e^{-2 k t}\right)\left(\sum_{m} a_{m}^{2}\right)=\infty .
\end{aligned}
$$

Therefore we conclude that $T_{t} X \cdot \notin \sigma_{2}\left(H, H_{-\beta}\right)$.

If the equation (5.1) has a solution in $H$, then the solution should be given by $X_{t}=T_{t} X_{0}+\int_{0}^{t} T_{t-s} X_{s} \cdot d B_{s}$. As was mentioned in the Remark 5.1, the integral $\int_{0}^{t} T_{t-s} X_{s} \cdot d B_{s}$ does not always converge in the space $H$. Suppose next that a solution $X_{t}$ were obtained in $H_{-\infty}$. Then, by Proposition 5.1 (iii), the integral $\int_{0}^{t} T_{t-s} X_{s} \cdot d B_{s}$ would not be defined in $H_{-\infty}$. Thus we have seen that the equation (5.1) has no solution in $H_{-\infty}$. Accordingly, we will consider a modified equation of the form

$$
d X_{t}=-\hat{\omega} X_{t} d t+f\left(X_{t}\right) \cdot d B_{t},
$$

where $f$ is a mapping from $H_{-\alpha}$ to $H(\alpha>1 / 2)$.

THEOREM 5.1. If the mapping $f: H_{-\alpha} \rightarrow H, \alpha>1 / 2$, is Lipschitz continuous, then the equation (5.4) has a unique solution in $H_{-\alpha}$ for a given initial data $X_{0} \in H_{-\alpha}$.

Proof. From Proposition 5.1 (ii) and the Lipschitz continuity of $f$, it follows that

$$
\begin{aligned}
\|f(X)-f(Y)\|_{\sigma_{2}\left(H, H_{-\alpha}\right)}^{2} & \leqq \text { const. }\left(1+\sum_{k=1}^{\infty} k^{-2 \alpha}\right)\|f(X)-f(Y)\|^{2} \\
& \leqq \text { const. }\|X-Y\|_{-\alpha}^{2} .
\end{aligned}
$$

Therefore $f(X)$. is a Lipschitz continuous mapping from $H_{-\alpha}$ to $\sigma_{2}\left(H, H_{-\alpha}\right)$. Using Theorem 2.3, we know the existence of a solution of (5.4) as well as uniqueness.

From now on we treat only such a special case as $f$ is linear and of the form

$$
f(X)(\sigma)=\int_{0}^{\pi} \Gamma\left(\sigma, \sigma^{\prime}\right) X\left(\sigma^{\prime}\right) d \sigma^{\prime}, \quad X \in H_{-1},
$$

where $\Gamma\left(\sigma, \sigma^{\prime}\right)$ is an element of $H \otimes H_{1}$. Now, by Theorem 2.1 and Theorem 2.2 , the equation (5.4) is equivalent to 


$$
X_{t}=T_{t} X_{0}+\int_{0}^{t} T_{t-s}\left(f\left(X_{s}\right) \cdot\right) d B_{s} .
$$

We are now ready to apply the results obtained in $\S 4$ in terms of the integral representation of (5.6).

LEMMA 5.1. Let $S \in L^{2}\left(T \times \mathscr{E}^{*} \rightarrow \sigma_{2}\left(H, H_{-1}\right)\right)$ and assume that $S(t, \omega)$ satisfies the following conditions:

(i) $S(t, \omega)$ is $\mathscr{B}_{t}$-adapted.

(ii) $S(t, \cdot) \in L^{2}\left(\mathscr{E}^{*} \rightarrow \sigma_{2}\left(H, H_{-1}\right)\right)$ and the Wiener's direct sum decomposition of $S(t)$ is given by

$$
\begin{aligned}
& S(t, \omega)=\sum S_{n}(t), S_{n}(t) \in \mathscr{H}_{n}\left(\sigma_{2}\left(H, H_{-1}\right)\right), \\
& S_{n}(t) \cong S_{n}\left(t ; x_{1}, \cdots, x_{n} ; \boldsymbol{x}\right), x_{j}=\left(\boldsymbol{x}_{j}, t_{j}\right) \in D \times T, x \in D,
\end{aligned}
$$

where $S_{n}(t ; \cdot, \cdots, \cdot ; \cdot) \in L^{2}\left((\widehat{D \times T})^{n} \times D \rightarrow H_{-1}\right), D=[0, \pi]$ and $T=[0, \infty)$.

Then the $\mathscr{H}_{n+1}\left(H_{-1}\right)$-component of $\int_{0}^{t} S(s) d B_{s}$ is equal to $\int_{0}^{t} S_{n}(s) d B_{s}$ and its kernel is given by

$$
\frac{1}{n+1} \chi_{[0, t]}\left(t_{n+1}\right) S_{n}\left(t_{n+1} ; x_{1}, \cdots, x ; \boldsymbol{x}_{n+1}\right), t_{1} \leqq t_{2} \leqq \cdots \leqq t_{n+1} .
$$

Proof. Using the results in $\S 4$, we get

$$
\begin{aligned}
\int_{0}^{t} S_{n}(s) d B_{s} & =\int_{0}^{t} n ! \hat{I}\left(S_{n}(s)\right) d B_{s} \\
& =n ! \int_{0 \leqq t_{1} \leqq \cdots \leqq t_{n+1} \leqq t} \int S_{n}\left(t_{n+1} ; x_{1}, \cdots, x_{n} ; \boldsymbol{x}_{n+1}\right) d B_{t_{1}}\left(\boldsymbol{x}_{1}\right) \cdots d B_{t_{n+1}}\left(\boldsymbol{x}_{n+1}\right) \\
& =(n+1) ! \hat{I}\left(\frac{1}{n+1} \chi_{[0, t]} S_{n}\left(t_{n+1} ; x_{1}, \cdots, x_{n} ; \boldsymbol{x}_{n+1}\right)\right) .
\end{aligned}
$$

LEMMA 5.2. Let $Z \in L^{2}\left(\mathscr{E}^{*} \rightarrow H\right)$ and let $Z_{n}\left(x_{1}, \cdots, x_{n} ; x\right), n=0,1,2, \cdots$, be the kernels of the integral representation of $Z$. Then the kernels $F_{n}\left(x_{1}, \cdots\right.$, $\left.x_{n} ; \boldsymbol{x}\right), n=0,1,2, \cdots$, of the integral representation of the multiplicative operator $Z \cdot \in L^{2}\left(\mathscr{E}^{*} \rightarrow \sigma_{2}\left(H, H_{-1}\right)\right)$ are of the form

$$
F_{n}\left(x_{1}, \cdots, x_{n} ; \boldsymbol{x}\right)=\sum_{j=0}^{\infty} Z_{n}\left(x_{1}, \cdots, x_{n} ; \boldsymbol{x}\right) \xi_{j}(\boldsymbol{x}) \xi_{j}, n=0,1,2, \cdots .
$$

Proof. Put $(Z)_{n}=\mathscr{H}_{n}(H)$-element of $Z$. Then $(Z)_{n}$ satisfies

$$
\left((Z)_{n} \xi, \xi_{j}\right)=\int_{0}^{\pi}(Z)_{n}(\sigma) \xi(\sigma) \xi_{j}(\sigma) d \sigma=\left((Z)_{n}, \xi \xi_{j}\right)
$$




$$
\begin{aligned}
& =\left(I\left(Z_{n}\right), \xi \xi_{\jmath}\right)=I\left(Z_{n}^{*} \xi \xi_{j}\right) \\
& =I\left(\int_{0}^{\pi} Z_{n}\left(x_{1}, \cdots, x_{n} ; \sigma\right) \xi(\sigma) \xi_{j}(\sigma) d \sigma\right) .
\end{aligned}
$$

Therefore we have

$$
(Z)_{n} \xi=I\left(\sum_{j=0}^{\infty}\left(\int_{0}^{\pi} Z_{n}\left(x_{1}, \cdots, x_{n} ; \sigma\right) \xi(\sigma) \xi_{j}(\sigma) d \sigma\right) \xi_{j}\right) .
$$

From this equality we obtain

$$
\begin{aligned}
(Z \xi)_{n} & =(Z)_{n} \xi=I\left(\sum_{j=0}^{\infty}\left(\int_{0}^{\pi} Z_{n}\left(x, \cdots, x_{n} ; \sigma\right) \xi(\sigma) \xi_{j}(\sigma) d \sigma\right) \xi_{j}\right) \\
& =I\left(\int_{0}^{\pi}\left\{\sum_{j=0}^{\infty} Z_{n}\left(x_{1}, \cdots, x_{n} ; \sigma\right) \xi_{j}(\sigma) \cdot \xi_{j}\right\} \xi(\sigma) d \sigma\right) .
\end{aligned}
$$

The equality (5.9) proves (5.8).

LEMmA 5.3. Let $Z \in L^{2}\left(T \times \mathscr{E}^{*} \rightarrow H\right)$ and let $Z_{n}\left(t ; x_{1}, \cdots, x_{n} ; x\right), n=$ $0,1,2, \cdots$, be the kernels of $Z$. Then the kernels of the operator $T_{t-s}(Z(s) \cdot)$ are of the form

$$
Z_{n}\left(s ; x_{1}, \cdots, x_{n} ; \boldsymbol{x}\right)\left\{\sum_{j=0}^{\infty} e^{-\jmath(t-s)} \xi_{j}(\boldsymbol{x}) \xi_{j}\right\} .
$$

Proof. From (5.9) it follows that

$$
\begin{aligned}
& T_{t-s}(Z(s) \cdot \xi)_{n} \\
& \quad=I\left(\int_{0}^{\pi}\left\{\sum_{j=0}^{\infty} Z_{n}\left(s ; x_{1}, \cdots, x_{n} ; \sigma\right) \xi_{j}(\sigma) e^{-j(t-s)} \xi_{j}\right\} \xi(\sigma) d \sigma\right) .
\end{aligned}
$$

This equality proves the lemma.

(Q.E.D.)

Lemma 5.4. Let $X_{s} \in L_{2}\left(\mathscr{E}^{*} \rightarrow H_{-1}\right)$ and let its kernels be $\Phi_{n}\left(s ; x_{1}, \cdots, x_{n}\right.$; $\left.\sigma^{\prime}\right), n=0,1,2, \cdots$. Then the kernels of the integral representation of $\int_{0}^{\pi} \Gamma\left(\sigma, \sigma^{\prime}\right) X_{s}\left(\sigma^{\prime}\right) d \sigma^{\prime}$ are expressed in the form

$$
\int_{0}^{\pi} \Gamma\left(\sigma, \sigma^{\prime}\right) \Phi_{n}\left(s ; x_{1}, \cdots, x_{n} ; \sigma^{\prime}\right) d \sigma^{\prime},
$$

where the integration should be understood in the same sense as in (5.5).

Proof. This lemma is obvious from the definition of kernels.

(Q.E.D.)

Corollary 5.1. Under the same assumptions as in Lemma 5.4, the kernels of the operator $T_{t-s}\left(f\left(X_{s}\right) \cdot\right)$ are given by 


$$
\int \Gamma\left(\boldsymbol{x}, \sigma^{\prime}\right) \Phi_{n}\left(s ; x_{1}, \cdots, x_{n} ; \sigma^{\prime}\right) d \sigma^{\prime} \sum_{j=0}^{\infty} e^{-j(t-s)} \xi_{j}(x) \xi_{j} .
$$

Proof. This comes from Lemma 5.3 and 5.4.

We are now in a position to describe the equation (5.6) in terms of the integral representation.

THEOREM 5.2. (i) Let $X_{t}$ be the solution of (5.6), where $f(X)=$ $\int_{0}^{\pi} \Gamma\left(\sigma, \sigma^{\prime}\right) X\left(\sigma^{\prime}\right) d \sigma^{\prime}$, and let $\Phi_{n}\left(t ; x_{1}, \cdots, x_{n} ; \cdot\right)$ be the kernels of the integral representation of $X_{t}$. Then $\Phi_{n}, n=0,1,2, \cdots$, satisfies the following functional equation

$$
\begin{aligned}
\Phi_{n+1}(t ; & \left.x_{1}, \cdots, x_{n+1} ; \cdot\right) \\
= & \frac{1}{n+1} \chi_{[0, t]}^{(n+1) \otimes}\left(t_{1}, \cdots, t_{n+1}\right)\left\{\sum_{j=0}^{\infty} e^{-j\left(t-t_{n+1}\right)} \xi_{j}\left(x_{n+1}\right) \xi_{j}\right\} \\
& \times \int_{0}^{\pi} \Gamma\left(x_{n+1}, \sigma^{\prime}\right) \Phi_{n}\left(t_{n+1} ; x_{1}, \cdots, x_{n} ; \sigma^{\prime}\right) d \sigma^{\prime} \\
& t_{1} \leqq t_{2} \leqq \cdots \leqq t_{n+1}, n=0,1,2, \cdots, \\
\Phi_{0}(t ; \cdot) & =T_{t} X_{0}
\end{aligned}
$$

(ii) Conversely, if a solution $\Phi_{n}\left(t ; x_{1}, \cdots, x_{n} ; \cdot\right), n=0,1,2, \cdots$, of the equation (5.13) satisfies the following condition

$$
\sum_{n=0}^{\infty}(n !)^{2} \int_{t_{1} \leqq t_{2} \leqq \cdots \leqq t_{n}} \cdots \int\left\|\Phi_{n}(t)\right\|_{-1}^{2} d x_{1} \cdots d x_{n}<\infty,
$$

then $\sum_{n=0}^{\infty} I\left(\Phi_{n}(t)\right)$ is the solution of (5.6).

Proof. (i) follows from Lemma 5.1 and Corollary 5.1. The condition (5.14) assures that $\sum_{n=0}^{\infty} I\left(\Phi_{n}(t)\right) \in L^{\prime}\left(\mathscr{E}^{*} \rightarrow \dot{H}_{-1}\right)$. Hence (ii) follows from the uniqueness of the integral representation.

(Q.E.D.)

TheOREM 5.3. The equation (5.13) has a unique solution for a given initial data $X_{0} \in H_{-1}$, and the solution $\Phi_{n}(t)$ itself satisfies

$$
(n !)^{2} \int_{t_{1} \leqq \cdots \leqq t_{n}} \cdots \int\left\|\Phi_{n}(t)\right\|_{-1}^{2} d x_{1} \cdots d x_{n} \leqq \frac{1}{n !} c_{0} c^{n} t^{n},
$$

where $c_{0}$ and $c$ are positive constants.

Remark 5.2. The condition (5.15) is equivalent to

$$
\left\|I\left(\Phi_{n}(t)\right)\right\|_{L^{2\left(\delta^{*} \rightarrow H-1\right)}}^{2} \leqq \frac{1}{n !} c_{0} c^{n} t^{n}
$$


Proof of Theorem 5.3. When a point $X_{0} \in H_{-1}$ is given, $\left\{\Phi_{n}(t) ; n=\right.$ $0,1,2, \cdots\}$ is determined inductively by (5.13). The proof of (5.15) proceeds by induction. Since $\left\|T_{t}\right\| \leqq 1, \Phi_{0}(t)$ satisfies (5.15) with $c_{0}=\left\|X_{0}\right\|_{-1}^{2}$. Let $c_{1}$ be a constant which satisfies

$$
\left\|\int \Gamma\left(\cdot, \sigma^{\prime}\right) X\left(\sigma^{\prime}\right) d \sigma^{\prime}\right\|^{2} \leqq c_{1}\|X\|_{-1}^{2} \quad \text { for any } X \in H_{-1} .
$$

Put $c=\left(1+\sum_{j=1}^{\infty}\left(1 / j^{2}\right)\right) c_{1}$. To prove the induction step from $n$ to $n+1$, we note that

$$
\begin{aligned}
((n+1) !)^{2} \int_{t_{1} \leqq \cdots \leqq t_{n+1}} \cdots \int\left\|\Phi_{n+1}(t)\right\|_{-1}^{2} d x_{1} \cdots d x_{n+1} \\
=(n !)^{2} \int_{0 \leqq t_{1} \leqq \cdots \leqq t_{n+1} \leqq t} \int\left\{\left\|\sum_{j=0}^{\infty} e^{-j\left(t-t_{n+1}\right)} \xi_{j}\left(x_{n+1}\right) \xi_{j}\right\|_{-1}^{2}\right\} \\
\quad \times\left\{\int_{0}^{\pi} \Gamma\left(x_{n+1}, \sigma^{\prime}\right) \Phi_{n}\left(t_{n+1} ; x_{1}, \cdots, x_{n} ; \sigma^{\prime}\right) d \sigma^{\prime}\right\}^{2} d x_{1} \cdots d x_{n+1} \\
\quad \leqq(n !)^{2} c \int_{0 \leqq t_{1} \leqq \cdots \leqq t_{n+1} \leqq t} \int\left\|\Phi_{n}\left(t_{n+1} ; x_{1}, \cdots, x_{n} ; \cdot\right)\right\|_{-1}^{2} d x_{1} \cdots d x_{n} d t_{n+1} \\
\quad \leqq \frac{1}{n !} c_{0} c^{n+1} \int_{0}^{t} t_{n+1}^{n} d t_{n+1}=\frac{1}{(n+1) !} c_{0} c^{n+1} t^{n+1} .
\end{aligned}
$$

Then, we have proved (5.15).

(Q.E.D.)

Corollary 5.2. The equation (5.4) has a unique solution $X_{t}$ under the assumption that $f(X)=\int_{0}^{\pi} \Gamma\left(\cdot, \sigma^{\prime}\right) X\left(\sigma^{\prime}\right) d \sigma^{\prime}$, and $X_{t}$ satisfies

$$
\left\|X_{t}\right\|_{L^{2}\left(\sigma^{*} \rightarrow H-1\right)}^{2} \leqq\left\|X_{0}\right\|_{-1}^{2} e^{c t} \text {. }
$$

Proof. From Theorem 5.2 and 5.3, it follows that $\sum_{n=0}^{\infty} I\left(\Phi_{n}(t)\right)$ is the unique solution of (5.4). The inequality (5.18) is obvious from (5.16).

(Q.E.D.)

Remark 5.3. The results in Corollary 5.2 are in fact part of Theorem 5.1 , because the estimation (5.18) can be obtained by successive approximation method.

Since $\Gamma\left(\sigma, \sigma^{\prime}\right)$ has been assumed to be an element of $H \otimes H_{1}, \Gamma\left(\sigma, \sigma^{\prime}\right)$ is expressed in the form of

$$
\begin{aligned}
& \Gamma\left(\sigma, \sigma^{\prime}\right)=\sum_{k, i=0}^{\infty} a_{k i} \xi_{k}(\sigma) \xi_{i}\left(\sigma^{\prime}\right), \\
& \|\Gamma\|_{H \otimes H_{1}}^{2}=\sum_{k=0}^{\infty} a_{k 0}^{2}+\sum_{k=0}^{\infty} \sum_{i=1}^{\infty} a_{k i}^{2} i^{2} .
\end{aligned}
$$


Then we have the following theorem.

THEOREM 5.4. When $\Gamma\left(\sigma, \sigma^{\prime}\right)$ is of the form (5.19), the unique solution of (5.13) is given by

$$
\begin{aligned}
\Phi_{0}(t)= & T_{t} X_{0}=\sum_{j=0}^{\infty} e^{-j t} c_{j} \xi_{j}, \\
\Phi_{n}\left(t ; x_{1}, \cdots, x_{n} ; \cdot\right) & \\
= & \frac{1}{n !} \chi_{[0, t]}^{n \otimes}\left(t_{1}, \cdots, t_{n}\right)\left[\sum_{j=0}^{\infty}\left\{\xi_{j}\left(\boldsymbol{x}_{n}\right) \exp \left\{-j\left(t-t_{n}\right)\right\} \xi_{j}(\cdot)\right\}\right. \\
& \times\left\{\sum_{\substack{k_{1}, \cdots, k_{n}=0 \\
i_{1}, \cdots, i_{n}=0}}^{\infty} a_{k_{n} i_{n}} \cdots a_{k_{1} i_{1}} \xi_{k_{n}}\left(\boldsymbol{x}_{n}\right) \exp \left\{-i_{n}\left(t_{n}-t_{n-1}\right)\right\} \xi_{i_{n}}\left(\boldsymbol{x}_{n-1}\right)\right. \\
& \left.\left.\times \cdots \times \xi_{k_{2}}\left(\boldsymbol{x}_{2}\right) \exp \left\{-i_{2}\left(t_{2}-t_{1}\right)\right\} \xi_{i_{2}}\left(\boldsymbol{x}_{1}\right) \xi_{k_{1}}\left(\boldsymbol{x}_{1}\right) \exp \left(-i_{1} t_{1}\right\} c_{i_{1}}\right\}\right], \\
& \quad t_{1} \leqq t_{2} \leqq \cdots \leqq t_{n}, \quad n=1,2, \cdots,
\end{aligned}
$$

where $X_{0}=\sum_{j=0}^{\infty} c_{j} \xi_{j} \in H_{-1}$ is a given initial value.

Proof. Using the expression (5.19), we are able to solve the equation (5.13) step by step in an explicit form. Carrying out this procedure, we can obtain the formula (5.19).

(Q.E.D.)

For $\eta \in \mathscr{E}$ put

$$
U^{(n)}(t ; \eta)=\int \cdots \int \Phi_{n}\left(t ; x_{1}, \cdots, x_{n} ; \cdot\right) \eta^{n \otimes}\left(x_{1}, \cdots, x_{n}\right) d x_{1} \cdots d x_{n},
$$

and put

$$
U(t ; \eta)=\sum_{n=0}^{\infty} U^{(n)}(t ; \eta)
$$

Then it is easily seen that the system $\{U(t ; \eta) ; \eta \in \mathscr{E}\}$ determines $\left\{\Phi_{n}(t\right.$; $\left.\left.x_{1}, \cdots, x_{n}\right), n=0,1,2, \cdots\right\}$ completely. Without loss of generality, we may assume that $\{\eta: \eta=\xi \otimes \zeta \in \mathscr{E}\}$ is dense in $\mathscr{E}$. We therefore conclude that $\{U(t ; \eta): \eta=\xi \otimes \zeta \in \mathscr{E}\}$ determines $\left\{\Phi_{n}(t) ; n=0,1,2, \cdots\right\}$ completely.

TheOREM 5.5. For $\eta=\xi \otimes \zeta \in \mathscr{E}, U(t ; \eta)$ satisfies the following equation

$$
\frac{d U(t ; \eta)}{d t}=-\hat{\omega} U(t ; \eta)+\zeta(t) G U(t ; \eta), \quad t>0, \quad U(0 ; \eta)=X_{0}
$$

where $G$ is a linear operator on $H_{-1}$ depending on $\Gamma$ and $\xi$ (the explicit form of $G$ is given in the proof).

Proof. Using (5.20), we get 


$$
\begin{aligned}
& U^{(n)}(t ; \eta) \\
& \quad=n ! \int_{t_{1} \leqq \cdots \leqq t n} \cdots \int \Phi_{n}\left(t ; x_{1}, \cdots, x_{n} ; \cdot\right) \eta^{n \otimes}\left(x_{1}, \cdots, x_{n}\right) d x_{1} \cdots d x_{n} \\
& \quad=\sum_{j=0}^{\infty} \xi_{j}\left[\sum a_{k_{n} i_{n}} \cdots a_{k_{1_{1}} i_{1}} c_{i_{1}} b_{j k_{n}} b_{i_{n} k_{n-1}} \cdots b_{i_{2} k_{1}} d_{\left(i_{1}, \cdots, i_{n}\right), j}^{(n)}(t)\right]
\end{aligned}
$$

where

$$
b_{i k}=\int_{0}^{\pi} \xi_{i}(\sigma) \xi_{k}(\sigma) \xi(\sigma) d \sigma, \quad i, k=0,1,2, \cdots,
$$

and

$$
\begin{aligned}
d_{\left(i_{1}, \cdots, i_{n}\right), j}^{(n)}(t)= & \int_{0 \leqq t_{1} \leqq \cdots \leqq t_{n} \leqq t} \int \exp \left\{-j\left(t-t_{n}\right)-\cdots-i_{1} t_{1}\right\} \\
& \times \zeta\left(t_{1}\right) \cdots \zeta\left(t_{n}\right) d t_{1} \cdots d t_{n}, \quad n=1,2, \cdots .
\end{aligned}
$$

From

$$
d_{\left(i_{1}, \cdots, i_{n}\right), j}^{(n)}(t)=\int_{0}^{t} \exp \left\{-j\left(t-t_{n}\right)\right\} \zeta\left(t_{n}\right) d_{\left(i_{1}, \cdots, i_{n-1}\right), i_{n}}^{(n-1)}\left(t_{n}\right) d t_{n},
$$

it follows that

$$
\begin{aligned}
& \frac{d}{d t} d_{\left(i_{1}, \cdots, i_{n}\right), j}^{(n)}(t) \\
& \quad=\zeta(t) d_{\left(i_{1}, \cdots, i_{n-1}\right), i_{n}}^{(n-1)}(t)-j d_{\left(i_{1}, \cdots, i_{n-1}, j\right.}^{(n-1)}(t), \quad n=1,2, \cdots,
\end{aligned}
$$

where $d^{(0)}(t)=0$.

From (5.24) and (5.25) we have

$$
\begin{aligned}
& \frac{d}{d t} U^{(n)}(t ; \eta) \\
&=-\hat{\omega} U^{(n)}(t ; \eta) \\
&+\zeta(t) \sum_{j=0}^{\infty} \xi_{j}\left[\sum a_{k_{n} i_{n}} \cdots a_{k_{1} i_{1}} c_{i_{1}} b_{j k_{n}} \cdots b_{i_{2} k_{1}} d_{\left(i_{1}, \cdots, i_{n-1}\right), i_{n}}^{(n-1)}(t)\right] .
\end{aligned}
$$

Introduce an operator $G$ on $H_{-1}$

$$
G\left(\sum_{p=0}^{\infty} \alpha_{p} \xi_{p}\right)=\sum_{j=0}^{\infty}\left(\sum_{p, q} b_{j q} a_{q p} \alpha_{p}\right) \xi_{j} .
$$

Then the second term of $(5.26)$ is equal to $\zeta(t) G U^{(n-1)}(t ; \eta)$. Thus we have

$$
\frac{d}{d t} U^{(n)}(t ; \eta)=-\hat{\omega} U^{(n)}(t ; \eta)+\zeta(t) G U^{(n-1)}(t ; \eta), \quad n=1,2, \cdots
$$

$$
\frac{d}{d t} U^{(0)}(t ; \eta)=-\hat{\omega} U^{(0)}(t ; \eta)
$$


Summing up (5.28) for $n=0,1,2, \cdots$, we obtain (5.23). The convergence of the series appeared above is guaranteed by Theorem 5.3. (Q.E.D.)

Remark 5.4. The operator $G$ is expressible as

$$
G=\tilde{G}(\xi) \Gamma
$$

where $\tilde{G}(\xi)$ is a bounded linear operator on $H$, linearly depending on $\xi$, and where $\Gamma$ is the bounded linear operator from $H_{-1}$ to $H$ given by (5.5)

\section{REFERENCES}

[1] A. V. Balakrishnan, Stochastic optimization theory in Hilbert spaces-1, Applied Mathematics and Optimization, 1, No. 2 (1974), 97-120.

[2] - Stochastic bilinear partial differential equations, Proc. U.S.-Italy Conference on Variable Structure Systems, Oregon, 1974.

[ 3 ] P. Billingsley, Convergence of probability measures, Wiley, 1968.

[4] R. F. Curtain and P. L. Falb, Stochastic differential equations in Hilbert space, J. Differential Equations, 10 (1971), 412-430.

[ 5 ] R. F. Curtain, A survey of infinite-dimensional filtering, SIAM Review, 17, No. 3 (1975), 395-411.

[6] - Estimation theory for abstract evolution equations excited by general white noise processes, SIAM J. Control and Optimization, 14, No. 6 (1976), 1124-1150.

[7] $\mathrm{Yu}$ L. Daletskii, Infinite dimensional elliptic operators and parabolic equations connected with them, Uspekhi. Math. Nauk t. 22 (1967).

[ 8 ] D. A. Dawson, Stochastic evolution equation, Mathematical Biosciences, 15 (1972), 287-316.

[9] - Stochastic evolution equations and related measure processes, J. Multivariate Analysis, 5 (1975), 1-55.

[10] - Spatially homogeneous random evolutoins, to appear.

[11] I. M. Gelfand and N. Ya. Vilenkin, Generalized functions, Vol.4, Academic Press, 1964.

[12] Z. Haba, Functional equations for extended hadrons, J. Math. Phys., 18, No. 11 (1977), 2133-2137.

[13] Z. Haba and J. Lukierski, Stochastic description of extended hadrons, to appear.

[14] T. Hida, Analysis of Brownian functionals, Carleton Math. Lecture Notes No. 13, 2nd ed. Ottawa, Canada, 1978.

[15] M. Kaku and K. Kikkawa, Field theory of relativistic strings. 1. Trees, Physical Review D, 10, No. 4 (1974), 1110-1133.

[16] I. Kubo, Hida calculus on Gaussian white noise, Nagoya Univ. Lecture Notes, 1979.

[17] H. H. Kuo, Gaussian measures in Banach spaces, Lecture Notes in Math., Vol. 463, Springer-Verlag, 1975.

[18] R. Marcus, Parabolic Itô equations, Trans. Amer. Math. Soc., 198 (1974), 177-190.

[19] Y. Miyahara, Stochastic differential equations in Hilbert space, "OIKONOMIKA" (Nagoya City University, Japan), 14, No. 1 (1977), 37-47.

[20] - Stability of linear stochastic differential equations in Hilbert space, in Information, Decision and Control in Dynamic Socio-Economics (ed. by H. Myoken), Bunshindo/Kinokuniya, Tokyo, 1978, 237-252.

[21] K. R. Parthasarathy, Probability measures on metric spaces, Academic Press, 1967. 
[22] C. Rebbi, Dual models and relativistic quantum strings, Physics Reports (Section C of Physics Letters), 12, No. 1 (1974), 1-73.

[23] A. Shimizu, Construction of a solution of linear stochastic evolution equations on a Hilbert space, in Proceedings of the International Symposium on Stochastic Differential Equations, Kyoto, 1976, 385-395.

[24] A. V. Skorohod, Integration in Hilbert Space, Springer-Verlag, 1974.

[25] Xia Dao-xing, Measure and integration theory on infinite-dimensional spaces, Academic Press, New York and London, 1972.

[26] M. Yor, Existence et unicité de diffusions à valeurs dans un espace de Hilbert, Annales de l'Institut Henri Poincaré, Section B, 10, No. 1 (1974), 55-58.

Nagoya City University 